\title{
LE ARTI DECORATIVE ALLE GRANDI ESPOSIZIONI MILANESI, 1881, 1894, 1906: UNO SGUARDO CRITICO
}

\author{
ORNELLA SELVAFOLTA $(*)$
}

SunTO. - Il saggio evidenzia il ruolo delle arti decorative nella cultura del progetto ottocentesco attraverso lo specchio delle grandi esposizioni tenutesi a Milano tra Otto e Novecento. Dall'Esposizione nazionale e artistica del 1881, passando attraverso le Esposizioni Riunite del 1894 fino all'Esposizione Internazionale del Sempione del 1906, le arti decorative, nella loro variegata casistica di oggetti, tecniche e materiali, hanno rappresentato parti assai rilevanti delle manifestazioni, spesso contribuendo in modo determinante alla loro riuscita e al loro successo economico. Tali eventi costituiscono quindi un buon campo di osservazione sia per le tipologie dei prodotti, sia per le posizioni dei più accreditati osservatori dell'epoca e consentono di riflettere su aspetti quali i rapporti fra tradizione e innovazione, i cambiamenti stilistici e l'evoluzione del gusto, la formazione e la professione, in un arco di tempo che si può considerare fondamentale per il rinnovamento del settore e le prefigurazioni del moderno design.

$$
* * *
$$

A BSTRACT. - Purpose of the essay is to highlight the role of the decorative arts in nineteenth-century design culture using the "looking glass" of the great Milan exhibitions held in the decades between the nineteenth and twentieth centuries. From the National and Artistic Exhibition of 1881, through the United Expositions of 1894, to the Sempione International Exhibition of 1906, decorative and/or applied arts, in their variety of products, materials and techniques, have represented substantial parts of the events, often contributing to their cultural and economic success. The above three major exhibitions are therefore significant research fields as regards the characteristics of the products and their critical appraisals, enabling to consider some significant aspects of their history, such as the relationship between tradition and innovation, theory and practice, stylistic changes and the evolution of taste, during a time span that can be considered seminal for the renewal of the decorative arts and the foreshadowing of modern design.

(*) Politecnico di Milano, Italia. E-mail: ornella.selvafolta@polimi.it 
Arti decorative, arti industriali, arti applicate, arti ornamentali, arti utili, arti minori: basterebbe forse riferirsi a questa variegata terminologia ricordata da Alfredo Melani nel 1886, per avere un'idea della molteplicità e ampiezza di temi che connotano il settore ${ }^{1}$. Delle numerose denominazioni Melani non si preoccupava più di tanto, considerandole distinzioni un po' gratuite (e in questo saggio si seguirà il suo indirizzo), ma reputava invece del massimo interesse il campo di azione che queste individuavano nelle diverse espressioni di materie, tecniche, oggetti. Melani fu non solo architetto e insegnante, ma anche un autore straordinariamente prolifico di manuali e repertori illustrati, contributi storici e riflessioni critiche che, nell'insieme, dimostrano come attorno alla questione della 'utilità dell'arte' ruotassero temi rilevanti dell'economia e della cultura attinenti la produzione e il mercato, il ruolo sociale del progetto, la tecnica e l'arte, la formazione e il lavoro².

Di questi ed altri aspetti le esposizioni sono state uno specchio tra i più rappresentativi oltre che occasioni irripetibili di osservazione, verifica e ragionamenti che, non raramente, hanno contribuito al formarsi di una nuova consapevolezza critica. A partire dalla Great Exbibition di Londra del 1851 quando le arti applicate avevano suscitato giudizi severi e avevano innescato una crescente attenzione per gli oggetti della vita

1 A. Melani, L'ornamento policromo nelle arti e nelle industrie artistiche, Milano, Hoepli, 1886, Introduzione, s.n.p.: "Si va disputando ancora intorno al nome da darsi a queste benedette Arti applicate all' industria o industriali: taluni vogliono chiamarle così per la ragione, essi osservano: che è un volere umiliare le arti a dire che possono essere industriali [...]; altri vogliono che si chiamino Industrie artistiche come l'abbiamo chiamate noi; altri ancora pretendono che si chiamino Arti decorative come se tutte le arti non fossero decorative. Se ci fosse permesso di dire netto il pensier nostro su tal proposito, francamente mostreremmo il desiderio che la gente laboriosa la quale s'occupa di tali quisquilie utilizzasse un po' meglio il suo tempo".

2 Per una riflessione critica su questi temi cfr. il contribuito sempre valido di F. Bologna, Dalle arti minori all'industrial design. Storia di una ideologia, Bari, Laterza, 1972. Tra le numerose pubblicazioni di Melani ricordo almeno: Decorazione e industrie artistiche, Milano, Vallardi, 1888-1889, 2 voll.; Dell'ornamento nell'architettura, Milano, Vallardi, 1900-1901, 3 voll.; L'arte nell'industria, Milano, Vallardi, s.d. [post 1910]. Sulla figura di Melani (1859-1928) cfr. Alfredo Melani e l'architettura moderna in Italia. Antologia critica (1882-1910), a cura di M.L. Scalvini e F. Mangone, Roma 1999, con bibliografia completa degli scritti; M.G. Maestrelli, Alfredo Melani. Architetto, storico e critico dell'architettura, Firenze, Pontecorboli, 2001. 
materiale, per i processi di produzione, per i metodi educativi e, non ultimo, per le manifestazioni del gusto in un'epoca in cui le aspettative di decoro, anziché passare in sottordine nella prospettiva industriale, si erano intensificate grazie ai meno costosi sistemi di fabbricazione e alle aumentate possibilità di consumo ${ }^{3}$. Londra con le sue migliaia di merci aveva pienamente evidenziato non solo l'importanza del settore, ma anche la necessità di una solida base culturale al fine di individuare riferimenti sicuri e fissare concetti utili a garantire sia la qualità dei prodotti sia la qualità della domanda (Figg. 1 e 2).

Si può solo ricordare Owen Jones e la sua Grammar of Ornament che, contro l'anarchia e le incongruenze del gusto, contro le indebite imitazioni degli stili del passato, proponeva un percorso nella storia dell'ornamento e dei diversi stili, inteso non tanto come una magnifica raccolta di modelli, quanto come una dimostrazione di principi costantemente presenti, tali da potere stabilire la struttura e le regole di una 'sintassi’ comune alla pluralità delle manifestazioni e delle espressioni linguistiche ${ }^{4}$ (Fig. 3).

Istanze simili non saranno estranee né al mondo culturale milanese, né alle esposizioni cittadine del 1881, del 1894 e del 1906 che, a loro volta, pur se inferiori per ampiezza e ambizioni alle più ampie edizioni universali di Londra e Parigi', si possono considerare osservatori privilegiati per valutare, tracciare bilanci e formulare progetti. Non è un caso del resto che sulle esposizioni abbiano riflettuto due figure tra le più

3 Cfr. le considerazioni di R. N. Wornum, The Exbibition as a Lesson in Taste in The Art Journal Illustrated Catalogue. The Industry of All Nations 1851, London, George Virtue, 1851, I-XXIII. Per una prospettiva a noi contemporanea cfr. E. Gombrich, The sense of Order. A Study in the Psychology of Decorative Arts, London, Phaidon, 1979.

4 Cfr. The Grammar of Ornament by Owen Jones, Illustrated by Examples from Various Styles of Ornament, London, Day and Son,1856, reprint: London, Studio Edition, 1989: cfr. in particolare il testo introduttivo: General Principles in the Arrangement of Form and Colour, in Architecture and the Decorative Arts, which are Advocated throughout this Work, 5-8. Sull'importanza di questo testo cfr. O. Selvafolta, Qualche osservazione su "The Grammar of Ornament" di Owen Jones, Londra 1856, in Architettura dell'Eclettismo. Studi storici, rilievo e restauro, teoria e prassi dell'architettura, a cura di L. Mozzoni e S. Santini, Napoli, Liguori Editore, 2012, 241-269.

5 Contributo indispensabile anche per capire le differenze tra i vari tipi di esposizione è L. Aimone, C. Olmo, Le esposizioni universali 1851-1900. Il progresso in scena, Torino, Allemandi, 1990. 
influenti e rappresentative della cultura del periodo nei versanti tecnico e architettonico-artistico: vale a dire l'ingegnere Giuseppe Colombo e l'architetto Camillo Boito ${ }^{6}$. Le loro osservazioni aiutano a delineare aspetti rilevanti del tema decorativo e contemporaneamente rivelano come le esposizioni milanesi costituissero un contesto favorevole ad esprimerne la complessità e la ricchezza.

L'interesse di Colombo partiva da una visione ampia, capace di connettere i contenuti tecnici e gli obiettivi economici con i valori estetici e i fenomeni del gusto: fin dalla prima Esposizione Nazionale tenutasi a Firenze nel 1861 quando sulle pagine del quotidiano "La Perseveranza" aveva scritto non solo di dispositivi e processi meccanici, ma anche delle "applicazioni dell'arte" passibili di impiego diretto nelle circostanze della vita, ed aveva espresso il proprio disappunto per la scarsa qualità di forme e disegni, per la mancanza di novità, i costi eccessivi e le lavorazioni imperfette che stavano vanificando il tradizionale primato storico-artistico italiano a favore di altri paesi, con gravissimi scompensi economici ${ }^{7}$.

Alle esposizioni universali di Londra nel 1862, a Parigi nel 1867, a Vienna nel 1873, e ancora Parigi nel 1878, Colombo continuerà a osservare sia le macchine e le industrie, sia i prodotti delle arti applicate, riconoscendo che entrambi i settori erano il portato della modernità̀ ${ }^{8}$ Così

6 Entrambi troppo noti per tentare anche un accenno biografico o bibliografico significativo: mi limito quindi a citare: G. Colombo., Industria e politica nella storia d'Italia. Scritti scelti: 1861-1916, a cura di C.G. Lacaita, Cariplo-Laterza, Bari 1985, con saggio introduttivo del curatore. Per Boito: cfr. C. Boito, Il nuovo e l'antico in architettura, a cura di M.A. Crippa, Milano, Jaca Book, 1988, con saggio introduttivo del curatore e bibliografia degli scritti; Camillo Boito. Un protagonista dell'Ottocento italiano, a cura di G. Zucconi, T. Serena, Venezia, Istituto Veneto di Scienze, Lettere ed Arti, 2002. Altri contributi verranno citati in modo pertinente al testo.

7 Per l'esposizione fiorentina cfr. La esposizione Italiana del 1861. Giornale con 190 incisioni e con gli atti ufficiali della Commissione, Firenze, Bettini, 1861. Gli articoli di G. Colombo, L'Esposizione italiana in Firenze, escono su "La Perseveranza" in ottobre e novembre 1861; si trovano ora in Id., Industria e politica, cit., 89-152.

8 Tra i suoi scritti più interessanti, cfr. L'esposizione internazionale di Parigi del 1867, in "Il Politecnico", s.V, vol. V, parte letterario-scientifica, fasc. I e fasc. III, gennaio-marzo 1868, ora in Id., Industria e politica, cit., 163-203. Si segnala inoltre che nel 1879 Colombo figura come direttore di "L'arte, l'industria e la meccanica all'Esposizione di Parigi del 1878", uscito nel 1878 in 15 fascicoli come supplemento alla rivista "L'arte e l'industria". 
che alla vigilia dell'"Esposizione nazionale artistica" di Milano del 1881, nel famoso saggio Milano industriale, apparso sul terzo volume di Mediolanum, egli dedicava uno spazio consistente anche alla produzione decorativa, considerandola in particolare sintonia con la cultura cittadina e sottolineando come il suo mandato fosse quello di "soddisfare le molte esigenze del vivere civile" che la "cresciuta agiatezza e il raffinamento culturale" avevano generato "in tutte le classi sociali". Osservazioni lucide che stabilivano l'importanza del rapporto tra il generale avanzamento economico e il delinearsi di un nuovo profilo funzionale e simbolico dei prodotti, tra l'espandersi dei consumi e l' ingentilirsi' dei comportamenti, delle aspettative e degli usi.

"L'arte industriale è la fonte della prosperità della Francia: Parigi e Lione creano la moda e s'impongono per essa coi loro prodotti", asseriva Colombo, rimarcando poi che per sopravvivere molte manifatture milanesi erano costrette ad adeguarsi ai loro modelli. Se i tessuti di lusso adottavano disegni lionesi, se i bronzi decorativi "passavano per merci parigine e venivano apprezzati ed acquistati come tali"; se più in generale le "maniere milanesi" si stavano diffondendo principalmente per la loro capacità di imitare le mode di Francia, significava che quel paese si era costruita una solida fama di arbitro del gusto anche grazie al sostegno delle sua tradizioni di qualità e prestigio, in armonia con un'educazione diffusa e con il rinnovamento di metodi, modelli, disegni ${ }^{10}$. E' necessario "promuovere il senso e l'intelligenza del bello", concludeva Colombo, e a questo scopo bisognava "creare maestranze che abbiano non solo l'abilità della mano, ma l'attitudine ad adattarsi facilmente alla varietà della produzione: in modo [...] che la versatilità e il buon gusto diventino qualità naturali, acquisite collo studio e coll'abitudine e trasmesse per eredità da una generazione all'altra" ${ }^{11}$.

Le sue posizioni non erano affatto lontane dagli orientamenti di Camillo Boito, straordinariamente attivo nella rivalutazione delle cosiddette "arti minori", attraverso la promozione e il sostegno alla loro didattica. Anche uno sguardo veloce ai suoi scritti o agli impegni di architetto e professore rivela infatti la costante attenzione a un set-

9 G. Colombo, Milano industriale, in Mediolanum, Milano, Vallardi, 1881, vol. III, ora in Id., Industria e politica, cit., 211.

10 Ibidem, 228, 230, 231, 235.

11 Ibidem, 237. 
tore che era parte strategica di un più generale progetto culturale, mirante a raggiungere il "governo di tutte le arti" secondo una "concezione piramidale' di reciproche correlazioni che poneva al vertice l'architettura e alla base le conoscenze elementari del disegno utile alle professioni.

Il coinvolgimento di Boito con le esposizioni milanesi è significativo a partire dall' "Esposizione Storica d'Arte Industriale" allestita nel 1874 al salone dei Giardini Pubblici, dove si esibivano oggetti antichi, provenienti per buona parte dalle collezioni private cittadine, con lo scopo di dimostrare come l'arte risultasse "lodevolmente associata all'industria, senza distinzione di età o di origine" ${ }^{12}$. L'iniziativa era pensata anche in anticipazione di un futuro "Museo d'Arte Industriale" che, pur senza esiti concreti, ebbe il merito di stimolare nell'ambiente lombardo un'inedita attenzione al valore delle arti e delle loro applicazioni ${ }^{13}$. In modo non dissimile da Colombo, Boito rilevava come nel passato arte e industria avevano operato in "lodevole associazione", mentre nel presente il loro rapporto virtuoso era andato perso a favore di una sostanziale incultura, di un eccessivo individualismo e di una malriposta "alterigia artistica" che in realtà "dava prova di vanitosa debolezza" ${ }^{14}$.

Simili giudizi si rafforzeranno in occasione dell'Esposizione nazionale del 1881 quando, invitato a tenere una conferenza sulle industrie artistiche e a misurarne "la bellezza", Boito non poteva nascondere la propria delusione $^{15}$ (Figg. 4 e 5). Il suo campo di osservazione comprendeva diver-

12 Cfr. Esposizione Storica d'Arte Industriale in Milano 1874. Catalogo generale, Milano, Treves, 1874 (s.n.p.).

13 Sul progetto di istituzione del futuro museo cfr. E. Bairati, Il museo d'arte industriale: il museo della città, in Milano fin de siécle e il caso Bagatti Valsecchi. Memoria e progetto per la metropoli italiana, a cura di C. Mozzarelli e R. Pavoni, Milano, Guerini, 1991, 47-58. Il progettato Museo d'Arte Industriale avrà vita come Museo Artistico Municipale, poi Civiche Raccolte d'Arte Applicata: cfr. C. Salsi, Le Civiche Raccolte d'Arte Applicata ed Incisioni, in Il Castello di Milano, Milano, Scheiwiller, 1996; E. Colle, Introduzione, in Museo d'Arti Applicate. Mobili e intagli lignei, Milano, Electa, 1996, 11-23.

14 C. Boito, La mostra storica d'arte industriale a Milano, in "Nuova Antologia", 27, 9, settembre 1874, 132-133. L'articolo riprende e amplia il testo della conferenza Le industrie artistiche. Conferenza del Prof. Camillo Boito tenuta il 17 settembre 1881, in Conferenze sulla Esposizione tenute per incarico di S.E. il Ministro di Agricoltura, Industria e Commercio, Milano, Hoepli, 1881, 21-41.

15 Ibidem, 23. 
si raggruppamenti: in primis le cosiddette "arti usuali" (con le "classi" dedicate a "vestimenti, mobili, oggetti di uso domestico"), poi il gruppo della "ceramica e vetraria", indi tutti quei settori dove le "industrie" (nell'accezione ottocentesca oscillante tra produzione su ampia scala, manifattura e a volte laboratorio artigiano), avevano 'collaborato' con l'arte. Non esisteva cioè una vera sezione di arti industriali o arti decorative, considerate troppo vaste e inclusive per rientrare nei progetti di ordinamento e nei sistemi classificatori che le esposizioni ottocentesche elaboravano ogni volta, spesso faticosamente e senza grandi successi ${ }^{16}$.

Nella conferenza Boito dichiarava di avere trovato scarsi motivi di soddisfazione tra i ferri battuti, le ceramiche, i vetri, i mobili, i merletti e ovunque la "grazia potesse trovare [...] il suo luogo". Persino le macchine, $i$ "rumorosi ordigni di ferro e di acciaio" che "servono a produrre il moto e a trasformarlo in mille diversi modi", mostravano una forma di bellezza che era intrinseca alla logica dei principi e alla sincerità delle funzioni ${ }^{17}$, mentre al converso la maggior parte degli oggetti pareva incapace di conciliare utilità e estetica, nonché di esprimere valori di congruenza tra il disegno e l'uso, tra il materiale e la costruzione, tra l'espressione stilistica e i principi della composizione. Se le macchine nel loro assetto limpido e disincantato quasi spontaneamente chiarivano tali correlazioni, le "industrie artistiche" non raramente le rendevano oscure, confondendole dietro un "sovrappiù" di disegno e di lavoro. Dominava un che di "soverchio", un "rigoglio" eccessivo, certamente stimolato dall'evento espositivo, dagli intenti pubblicitari, dalla necessità di distinguersi entro uno scenario sovrabbondante di offerte, ma anche dalle tendenze diffuse di un gusto ineducato per cui si era operato quasi un ribaltamento nell'equilibrio tecnico e estetico degli oggetti, lontano dalla "bellezza e dalla grazia" quanto dal più semplice raziocinio.

Lasciando per ora lo sguardo esclusivo di Boito e ricorrendo all'abbondante pubblicistica che accompagnò la rassegna del 1881, emerge la

16 Cfr. in Relazione generale compilata dall'ing. Amabile Terruggia, segretario generale e compilata per cura del Comitato esecutivo dell'Esposizione, Milano, Bernardoni, 1883, 22: "La materia dell'ordinamento delle Esposizioni ha sempre affaticate le menti degli economisti e dei pratici attratti a vicenda dal duplice intento di rispettare la classificazione ed i graduali sviluppi delle industrie [...], non senza avere il dovuto riguardo nella disposizione delle cose [...]".

17 Le industrie artistiche. Conferenza, cit., 24. 
grande attenzione dedicata al settore dei mobili che, superando i 400 espositori, costituiva una delle "classi" più rappresentative: sintomo "dell'immenso sviluppo che questa industria continua ad avere in Italia" 18 . Diversa era tuttavia la valutazione sul livello di qualità: piuttosto negativa sul fronte dei mobili usuali e invece abbastanza favorevole sul fronte dei mobili di pregio e, soprattutto, di quelli in stile neorinascimentale che sembravano meglio esemplificare l'abilità e l'"attitudine [...] distinta e propria" del paese ${ }^{19}$. Lo aveva affermato già nel 1870 , in un resoconto comparato sulle "produzioni artistico-industriali" d'Europa, Jakob von Falke, autorevole storico e teorico, membro del corpo direttivo dell'Österreichisches Museum für Kunst und Industrie di Vienna, secondo cui l'Italia, grazie alle sue prestigiose tradizioni, non aveva rivali "nell'artistica lavorazione del legno" e nella maestria con cui imitava i mirabili modelli "del primo Rinascimento e del periodo raffaellesco [...]"20.

Per tutti gli anni Settanta questa era stata infatti una specifica virtù dell'ebanisteria italiana e in particolar modo dell'ebanisteria toscana dove rifulgevano i nomi di Luigi Frullini, di Egisto Gajani o dei Barbetti, la cui produzione aveva ottenuto premi e riconoscimenti nelle maggiori esposizioni internazionali $i^{21}$. Non innovativi dal punto di vista del disegno e dei metodi di fabbricazione, i loro mobili eccellevano in qualità e autorevolezza mantenendo in vita esperienze, idee e tecniche di un Rinascimento universalmente riconosciuto come il periodo più aureo nella storia delle arti e dei mestieri. Come tale alimentava un florido mercato delle esportazioni (di 263.000 lire nel 1865 e di ben 1.364 .000 lire nel 1878), grazie alle richieste dell'aristocrazia europea, della nuova ricca clientela d'oltreoceano, dei prestigiosi musei di arti applicate europei per i quali prodotti di questo tipo assolvevano a importanti fini didattici ed espositivi ${ }^{22}$.

Il loro successo riposava sulla correttezza del disegno, sulla lavo-

18 Relazione generale, cit., 74

19 G. Falke [J. von Falke], Prospetto comparato delle odierne produzioni artisticoindustriali nei moderni paesi colti, in "Guida per le arti e mestieri", 2, 5, 1870, 66.

20 Ibidem.

21 Per questo aspetto della produzione di mobili cfr. O. Selvafolta, 1850-1900, in A Ponte, C. Paolini, O. Selvafolta, Il bello ritrovato. Gusto, ambienti, mobili dell'Ottocento, Novara, De Agostini, 1990, 454-467. Per gli ebanisti citati cfr. A. De Gubernatis, Dizionario degli artisti italiani viventi. Pittori, scultori e architetti, Firenze, Le Monnier, 1889, ad vocem.

22 Ibidem, 459-461. Per la fortuna del Neorinascimento cfr. Reviving the 
razione sopraffina, sulla modellazione scultorea degli intagli e sulla ripresa di uno stile che, quale prima esplicita cultura di rivisitazione del passato, si sintonizzava con lo storicismo ottocentesco e poteva anche legittimarsi nella ragione politica del giovane paese italiano fino a diventare una sorta di espressione preferenziale dell'ideologia unitaria.

Non meraviglia quindi che i mobili d'impronta rinascimentale fossero abbondantemente presenti all'Esposizione del 1881, meno esclusivi in termini di coerenza storico-stilistica rispetto a quelli degli ebanisti toscani, ma altrettanto rappresentativi del gusto. I lombardi Luigi Moretti, Adriano Brambilla, Francesco Bortolotti, Pietro Zaneletti ${ }^{23}$ presentavano infatti "mobili artistici" con una speciale predilezione per la tipologia dello stipo, uno degli arredi più tipici del Rinascimento che offriva "largo campo alla decorazione" e consentiva di cimentarsi in composizioni complesse di forme, materiali, tecniche ${ }^{24}$. Si trattava cioè di un genere di mobile particolarmente adatto alle esposizioni dove l'artefice poteva mostrare doti di costruttore e ornatista, di "scultore e architetto", dovendo padroneggiare l'articolazione dei volumi, il bilanciamento tra pieni e vuoti, la ripartizione delle superfici con paraste, lesene, mensole, colonne, capitelli e potendo altresì inserire ornati e figure che arricchivano di 'racconti' ogni parte e ogni dettaglio (Fig. 6).

In una credenza di Zaneletti, gli sguardi 'avidi' di immagini dei visitatori ottocenteschi potevano così scoprire che le quattro cariatidi a sostegno del "cornicione" rappresentavano "le quattro stagioni", mentre le figure in bassorilievi sulle superfici di ante e specchiature trasportavano "nell'impero del mare dove signoreggia Nettuno che sopra un cavallo marino, passa in mezzo ai flutti agitati brandendo il terribile tridente"25 (Fig. 7). Altri mobili di analoga esuberanza guadagnavano

Renaissance in the Second Half of Nineteenth Century Italy, a cura di R. Pavoni, Cambridge-London, Cambridge University Press, 1997, e con maggiore attinenza ai temi di questo saggio: O. Selvafolta, The Legacy of the Renaissance in Nineteenth Century Architecture and Applied Arts Periodicals, 15-62; A. Zanni, The NeoRenaissance as the Image of the Private, 126-148.

23 Notizie sugli ebanisti citati in A. De Gubernatis, Dizionario degli Artisti italiani viventi. Pittori, scultori e architetti, Firenze, Le Monnier, 1889, ad vocem.

24 F. Ewerbeck, Dei lavori in legno del Medio Evo e del Rinascimento, in "Guida per le arti e mestieri”, nuova serie, vol. IV, 1875, 129.

25 Armadio di Pietro Zaneletti, in L'Esposizione italiana del 1881 in Milano illustrata, Milano, Sonzogno, 1881, 139. 
descrizioni entusiaste sui cataloghi, ma molti rappresentavano per Boito virtuosismi senza ragione e senza costrutto, del tutto lontani dall'idea di ornamento in connessione con l'uso nei suoi valori pratici e simbolici. Con la consueta verve egli accennava quindi a una "grande tavola portata da un gruppo di cariatidi o telamoni" che "v'ammaccano gli stinchi", o a "una cornice" con un "viluppo di puttini scolpiti" che si aiutano l'un l'altro a salire, concepita addirittura con il titolo: "l'unione fa la forza" ${ }^{26}$ : sintomi del sovrappiù e del "rigoglio" eccessivo di decorazione e significati (Figg. 8 e 9).

A queste ed altre produzioni era necessaria una drastica revisione culturale che doveva passare attraverso il miglioramento dell'insegnamento del disegno ai vari livelli del progetto, l'arricchimento dei "musei d'arte industriale nelle grandi città" e il coinvolgimento degli artisti nei processi produttivi, facendo quindi dell'esposizione del 1881 un'opportunità di miglioramento, sull'esempio di quella londinese ${ }^{27}$.

Non a caso tra il 1881 e il 1882 Boito pubblicava tempestivamente per i tipi della Hoepli il libro I principi del disegno e gli stili dell'ornamento, per stabilire "i fondamenti pedagogici" o, si potrebbe dire "la grammatica dell'espressione" 28 . "Alfabeto di ogni lavoro", "parola dell'operaio", "chiave di volta dell'educazione", al disegno spettava il ruolo fondamentale di guida nel complesso intreccio fra tecnica e arte, fra idea e realizzazione. Abbinata a I principi era la raccolta di tavole Ornamenti di tutti gli stili classificati in ordine storico, che ripercorrevano lo svolgersi dell'ornato nelle varie epoche e componevano un repertorio di modelli eccellenti per gusto e cultura, esteso dai dettagli architettonici ai ferri battuti, dai mobili alle stoffe, dalle pietre lavorate ai legni intagliati: una sorta di nuova "grammatica dell'ornamento" che entrò nella maggior parte delle biblioteche scolastiche, nei laboratori artigiani e negli studi degli architetti qualificandone la cultura visiva al di là delle divaricazioni dei mestieri' ${ }^{29}$ (Figg. 10 e 11).

26 C. Boito, Le industrie artistiche, cit., 27.

27 Ibidem, 39-41.

28 C. Boito, I principi del disegno e gli stili dell'ornamento, Milano, Hoepli, 1882. La fortuna editoriale del manuale è testimoniata dalle 7 edizioni che si succedettero dal 1882 al 1925; l'ultima ristampa, sempre per i tipi della Hoepli a Milano, è del 1988.

29 Le citazioni sono riprese da F., I principi del disegno e gli stili dell'ornamento. A un maestro novello mandandogli le 303 tavole dell'opera "Ornamenti di tutti di stili", lettere di Camillo Boito, in "L'ingegneria civile e le arti industriali", n. 7, luglio 1882, 
Tredici anni dopo, alle Esposizioni Riunite del 1894 allestite per la prima volta al Parco Sempione, lo scenario delle arti decorative non sembrava molto più promettente ${ }^{30}$. Del resto il programma, comprendente 11 mostre diverse, non accordava loro uno spazio e un ruolo specifici. Mobili, suppellettili e una congerie di prodotti assimilabili al mondo delle arti applicate comparivano in più sezioni e nelle innumerevoli classi della cosiddetta "esposizione internazionale operaia" che, "per la prima volta", intendeva raccogliere "con intenti scientifici" "gli umili lavori e le poderose organizzazioni, mostrando con sincerità gli elementi del problema sociale, che vuol essere risolto in pace col benefico concorso di tutti”' ${ }^{31}$ (Fig. 12).

Così il Manifesto del Comitato per le Esposizioni Riunite sintetizzava i temi pregnanti che scuotevano la Milano degli anni Novanta, auspicando che il concetto di concordia sociale fornisse un legante non solo al mondo complesso dei rapporti di lavori, ma anche alla complessità della manifestazione espositiva e di una "mostra operaja" eterogenea e vastissima. Ufficialmente non potevano partecipare alla mostra le industrie o le manifatture grandi, medie e piccole, ma solo i 'dipendenti' con i risultati del loro lavoro sia individuale sia collettivo: di fatto un escamotage che consentì a parecchie ditte di essere presenti all'esposizione sotto la comoda espressione di "operai della ditta ...", o con la semplice pubblicità dell'insegna commerciale.

Sparse tra una galleria e l'altra, le applicazioni dell'arte (arredi, ferri battuti, ceramiche e porcellane, metalli, vetri, pietre, avori e tessuti) componevano un quadro che meritò giudizi benevoli nei confronti degli sforzi individuali e invece valutazioni più severe riguardo ai concetti generali e alla struttura complessiva degli oggetti. Anche in quest'occa-

109. La raccolta di tavole è: Ornamenti di tutti gli stili classificati in ordine storico, Hoepli, Milano 1881; e Stoffe, intarsi ed altri ornamenti piani... appendice all'opera Ornamenti di tutti gli stili classificati in ordine storico, Milano, Hoepli, 1881, cfr. l'edizione economica in 2 voll., Ornamenti di vari stili e Stoffe, intarsi ed altri ornamenti piani, Milano, Hoepli, s.d. [ma in prima edizione 1895].

30 Cfr. Le Esposizioni Riunite di Milano 1894. Sport-Belle Arti-FotograficaOperaja-Teatrale-Filatelica-Geografica-Arti Grafiche-Pubblicità-Vini e Olii-Orticola, Milano, Sonzogno, 1894. Cfr. anche Milano 1894. Le esposizioni Riunite, a cura di R. Pavoni, O. Selvafolta, Milano, Camera di Commercio Industria Artigianato e Agricoltura di Milano - Cinisello Balsamo, Amilcare Pizzi, 1994.

31 Cfr. Il Manifesto del Comitato delle Esposizioni Riunite di Milano 1894, in Ibidem, 130. 
sione le produzioni di maggior pregio si attestavano sulla ripresa degli stili storici, in composizioni spesso corrette e in esecuzioni ben fatte, ma di scarsa creatività (Fig. 13). Insomma, negli anni Novanta, quando il panorama europeo si stava ormai da tempo interrogando su come rinnovare modi e linguaggi, alle Esposizioni Riunite che pur affrontavano ambiti moderni come la pubblicità, la fotografia o la previdenza, le arti decorative mantenevano stretti legami con i modelli tradizionali, aderendo ad una fase dello storicismo più disciplinata rispetto ai decenni precedenti, ma anche meno sperimentale e meno indipendente.

Tra i premiati nella classe "Ceramica e vetraria" figuravano "lavori ripresi dall'antico", come i bei vasi e calici nella tradizione veneziana del XVI secolo presentati dagli operai delle vetrerie di Murano, o le ceramiche con morbide curve tra barocco e rococò esibite dagli operai della manifattura Antonibon di Nove vicino a Bassano, forte di due secoli di attività e di molti riconoscimenti ottenuti internazionalmente per la produzione di vasi, piatti, figurette "nel carattere primitivo dello stile barocco" ${ }^{22}$ (Fig. 14).

Sintomatico anche il successo ottenuto dalle sale neorinascimentali presentate dagli Artefici Associati e dai Fratelli Mora: questi ultimi ebanisti e arredatori originari di Bergamo, che a Milano avevano impiantato una fiorente attività di produzione e commercio di arredi nello stile tra Cinque e Seicento e avevano allestito un museo di pezzi antichi da cui i clienti potevano scegliere e ottenere fedeli riproduzioni $^{33}$. Il salotto presentato nel 1894 era sontuoso e autorevole, con mobili in "puro stile Rinascenza", ricchi di intagli, tappezzerie damascate e rivestimenti in cuoio istoriato. Proprio il cuoio e la sua pregiatissima lavorazione ad alto e basso rilievo, a imitazione degli antichi prodotti di Cordova, di Venezia e della Fiandra, realizzata in base a uno speciale brevetto, suscitava il massimo appezzamento. La mostra dei Mora era indipendente da quella operaia e non partecipava ai premi per la sua riconosciuta eccellenza, ma non di meno era una dimostrazione di come il rifarsi alla storia e replicare i modelli del passato fosse ancora un vessillo di qualità e prestigio (Fig. 15).

32 Oltre al catalogo Le Esposizioni Riunite di Milano 1894, cit. cfr. R. Pavoni, L'Esposizione operaia e le arti decorative, in Milano 1894, cit., 57-64 e Id., Il Magazzino generale del mobilio, in Ibidem, 63-68.

33 Cfr. O Selvafolta, I Fratelli Mora e il Museo d'Arte Antica, in C. Paolini, A. Ponte, O. Selvafolta, Il bello ritrovato, cit., 466; R. Pavoni, Il Salone Mora, in Milano 1894, cit., 105-108. 
Il vero cambiamento si ebbe con l'Esposizione Internazionale del Sempione del 1906: una straordinaria ribalta per suggellare il primato economico della città e proiettarla verso un orizzonte internazionale $^{34}$. Nata in seguito al traforo ferroviario del Sempione e originariamente incentrata sul tema dei trasporti e delle comunicazioni, l'esposizione andò presto ampliandosi fino ad includere numerose altre sezioni: l'Architettura e l'Agraria, la Previdenza e l'Igiene, le Belle Arti e, per l'appunto, le Arti Decorative. Per la prima volta presenti a Milano con questa denominazione, esse comprendevano "pittura e plastica ornamentale, vetri, mosaici e infissi di edifici, stoffe, metalli, rilegature, abbigliamento, arredamenti completi di appartamenti privati, di lusso ed economici, restaurants, birrerie, caffè, case operaie, ecc.": il tutto moltiplicato per i diversi paesi, tanto da rappresentare nell'insieme la parte più ampia dell'intera rassegna dopo quella dei trasporti $i^{35}$. E, non diversamente da prima, il settore era ancora rappresentativo di processi in divenire nel confronto con i numerosi problemi imposti dalla modernità e dai suoi rinnovati orizzonti tecnici e artistici (Fig. 16).

A monte stava l'Esposizione Internazionale di Arte Decorativa Moderna di Torino del 1902 dedicata alle "manifestazioni artistiche e ai prodotti industriali" inerenti "l'estetica della via, della casa e della stanza"36: manifestazione che aveva posto in primo piano questioni

34 Tra i principali cataloghi illustrati dell'esposizione: Milano e l'Esposizione Internazionale del Sempione 1906. Cronaca illustrata dell'Esposizione, a cura di E.A. Marescotti e Ed. Ximenes, Milano, Treves, 1906; L'Esposizione illustrata di Milano del 1906. Giornale Ufficiale del Comitato Esecutivo, Milano, Sonzogno, 1906; L'Esposizione di Milano 1906, Bergamo, Pro Familia, 1906. Tra i contributi recenti: Milano verso il Sempione, a cura di R. Cordani, Milano, Celip, 2006; La scienza, la città, la vita. Milano 1906: L'Esposizione internazionale del Sempione, a cura di P. Redondi e D. Lini, Milano, Skira, 2006; Milano e l'Esposizione internazionale del 1906. La rappresentazione della modernità, a cura di P. Audenino et al., Milano, FrancoAngeli, 2008.

35 Per le arti decorative cfr. O. Selvafolta, Milano 1906. L'Esposizione Internazionale del Sempione e le arti decorative al "principio di un'epoca nuova", Milano, Comune di Milano, Biblioteca d'Arte, 2009. La citazione è tratta da: Il Programma della Sezione dell'Arte Decorativa, in Milano e l'Esposizione Internazionale, cit., 55-56.

36 Esposizione d'Arte Decorativa Moderna. Regolamento generale, Torino 1901, cit. da Torino 1902 polemiche in Italia sull'Arte Nuova, a cura di F.R. Fratini, Torino, Martano, 1970, 134. Su questa mostra cfr. il contributo più esaustivo e recente La nascita del Liberty. Torino 1902, a cura di R. Bossaglia, E. Godoli, M. Rosci, Milano, Bompiani, 1996. 
moderne non tanto o non solo come ipotesi di rinnovamento formale, ma anche come necessità di rispondere con mezzi adeguati ai bisogni dell'oggi. In quell'occasione l'Italia aveva "ufficialmente" aderito al modernismo europeo nelle sue varie declinazioni di Liberty, Art Nouveau, Secession, Jugendstil..., guadagnando in visibilità e reputazione per essere stato il primo paese ad organizzare una rassegna internazionale destinata alle sole arti decorative e per aver adottato un regolamento che aveva escluso "le imitazioni degli stili del passato" e aveva ammesso solo "prodotti originali" e dimostrativi di "una decisa tendenza al rinnovamento" ${ }^{37}$.

Dopo quattro anni Milano intendeva consolidare tale indirizzo, ma la distanza temporale troppo ravvicinata, le rapide oscillazioni del gusto e le non infondate considerazioni che buona parte della produzione nazionale traeva ancora alimento dalla grandezza del suo passato, fecero adottare un indirizzo meno intransigente e più sfumato. Si esclusero quindi "le servili imitazioni del passato", ma non si bandirono le creazioni cosiddette retrospettive, ispirate ai "tesori di bellezza [...] accumulatisi nei secoli", purchè dotate di coerenza tecnica, funzionale, estetica: si accettarono cioè anche prodotti di gusto più tradizionale, a volte in precario equilibrio sul sottile crinale che, in quegli anni, ancora divideva l'invenzione dalla copia, la novità dalla rielaborazione di esempi preesistenti, le scelte moderne da quelle conservatrici ${ }^{38}$.

Lo stesso "palazzo dell'arte decorativa", firmato dall'architetto Sebastiano Locati, al Parco Sempione, era un'ibridazione tra passato e presente che, pur escludendo la replica in stile, adottava un'esuberanza linguistica più affine alle curve e alla plastica barocca che al nervoso coup de fuet Art Nouveau o ai linearismi della Secessione. (Fig. 17) Un "bislacco amalgama di antico e moderno", osservava il critico d'arte Vittorio Pica che, in una serie di articoli sulla rivista "Emporium", lamentava anche i criteri troppo mercantili sottostanti ad alcune scelte,

37 Torino 1902, cit., 134.

38 Il Programma della Sezione, cit., pp. 55-56. La Commissione per l'Arte decorativa era composta dall'arch. Gaetano Moretti presidente, arch. Giovanni Battista Borsani, vice-presidente, prof. Luigi Cavenaghi, vice-presidente; arch. Antonio Carminati, arch. Luigi Conconi, scultore Bassano Danielli, Luigi Della Torre, ing. Emilio Gussalli, prof. Lodovico Pogliaghi, Riccardo Salvadori, Giuseppe Vallardi. 
il graduale prevalere delle ragioni "degli affari" su quelle "della cultura e dell'educazione", la stessa ambiguità del programma oscillante tra passato e presente ${ }^{39}$.

Traspare dai suoi scritti, così come da quelli di altri osservatori meno inclini alla celebrazione retorica, il senso di una promessa mancata e di un rinnovamento interrotto che, d'altro canto, rispecchiano bene gli indirizzi culturali dell'epoca, non insensibili alle richieste del presente e neppure al proprio retaggio storico-artistico: segni di legami complessi e profondi tra la storia e la contemporaneità, o meglio, per usare una definizione di Luca Beltrami, tra il "ricordo del passato" e "l'intuito dell'avvenire" ${ }^{40}$. Tale orientamento è stato per certi versi responsabile (e in parte ancora lo è) dell'insuccesso storico-critico che ha investito molta parte della produzione decorativa e architettonica del periodo e ha spesso trascurato di rilevarne la dignità di approccio, la padronanza del disegno, la cura esecutiva, il substrato culturale: valori che al di là degli stili, sapranno sopravvivere nel tempo come una specifica e apprezzata attitudine nazionale, capace anche di innestarsi nelle forme e nei modi del moderno design.

Tornando tuttavia alle arti decorative nel 1906, è giusto sottolineare che la loro vastità (superavano l'intera rassegna di Torino), la grande partecipazione degli espositori e l'afflusso di pubblico ne decretarono il successo, mentre la dimensione internazionale e la varietà delle campionature riuscirono ad offrire uno spaccato significativo della produzione europea. Organizzate in rami merceologici, in sequenze di "ambienti completi", oppure come comparti di singoli padiglioni, le arti decorative si trovavano nelle due diverse sedi dell'esposizione: nel parco Sempione il già citato "palazzo dell'Arte Decorativa" (20.000 $\mathrm{mq}$ ), ospitava principalmente l'Italia e l'Ungheria e, in spazi più piccoli,

39 Il Palazzo dell'Arte Decorativa, in Milano e l'Esposizione Internazionale del Sempione 1906. Cronaca, cit. 118. Tra gli scritti di V. Pica sulle arti decorative all'esposizione cfr. la serie di articoli L'arte decorativa all'Esposizione di Milano, in "Emporium", 24, 1906: Il padiglione belga, n. 139, luglio, 2-20; La sezione ungherese, $\mathrm{n}$. 140, agosto, pp. 83-102; Qua e là per le sezioni straniere, n.141, settembre, 163-180; La Sezione Italiana I, n. 142, ottobre 1906, 244-255; La Sezione Italiana II, n. 143, novembre 1906, 323-340.

40 L. Beltrami, La mostra della ditta Ceruti (architetto Gaetano Moretti) all'Esposizione d'Arte Decorativa Moderna di Torino, in «L'Edilizia Moderna», a. XII, maggio 1903, 31. 
l'Olanda, l'Inghilterra, la Cina, il Giappone; in piazza d'Armi la Francia, forte della sua allure e del consolidato prestigio internazionale, disponeva di un padiglione esclusivo di $10.000 \mathrm{mq}$; mentre i prodotti del Belgio, della Russia, della Germania e dell'Austria occupavano parte dei rispettivi edifici nazionali ${ }^{41}$.

Partendo da quest'ultima sede e dovendo operare una drastica selezione tra i numerosissimi partecipanti, si segnala quindi la Francia, "sovranamente" installata nel proprio padiglione (architetto Orsino Bongi), che esibiva una vasta campionatura di prodotti riferiti per lo più al mondo della moda e a Parigi quale "novella Atene, regina del buon gusto e dell'artistica raffinatezza"42. "Più di tutto" vi erano raccolti "come in un colossale bazar" profumi e bijoux, "scarpette, fiori finti, nastri e cappellini", "tessuti, vesti e accessori del vestito" ${ }^{43}$. Non mancavano mobili, vetri e ceramiche dotati di "certa decorosa grazia", ma erano assenti i grandi nomi di Lalique o di Gallé, le stampe colorate di Grasset e i bei mobili intarsiati di Majorelle": ovvero gli artisti e i protagonisti dell'Art Nouveau che erano stati invece ammirati a Torino nel 1902. Privilegiando il lato commerciale, si era penalizzata la ricerca e la qualità, ma non si poteva negare che la mostra francese attirasse come un magnete e che i suoi espositori fossero maestri nell'arte del disporre, valorizzare e vendere gli oggetti secondo una concezione di mostra che aveva ormai abbandonato l'idea di rassegna universale a favore di obiettivi più marcatamente merceologici.

Più interessante sembrava ai critici la mostra di arte decorativa belga che, dentro al padiglione nazionale in stile tradizionale fiammingo, aveva allestito una parte moderna con sale curate da Victor Horta, Oscar van de Voorde, Léon Sneyers, Georges Hobé: architetti e decoratori per eccellenza, protagonisti 'storici' dell'Art Nouveau. Negli ambienti presentati a Milano avevano saputo concertare gli arredi e le suppellettili con dipinti e sculture, ovvero "l'arte applicata con l'arte

41 A queste sedi si aggiungevano poi i padiglioni di istituzioni variamente coinvolte nel mondo delle arti decorative (ad esempio la Società Umanitaria), di settori specifici come quello dell'oreficeria, nonchè di aziende che presentavano individualmente i loro prodotti (notevole successo riscosse la ditta Volpi di Udine). Cfr. infra i cataloghi citati nella nota 34 .

42 La Francia e il Belgio, in L'Esposizione di Milano 1906, cit., 57.

43 Ibidem, p. 58. Cfr. anche Il Padiglione francese, in L'Esposizione illustrata di Milano, cit., 316. 
pura", materializzando il programma ideale di fusione tra le arti che, in quello scorcio di secolo, rappresentava una delle più sentite istanze innovatrici ${ }^{44}$ (Figg. 18 e 19).

In linea con le motivazioni originarie dell'esposizione, legate ai trasporti, l'Austria aveva invece ideato un padiglione simile a una stazione ferroviaria (architetto Ludwig Baumann) dove la parte dei binari alloggiava vagoni e locomotive e l'edificio viaggiatori si componeva di ambienti con arredi e oggetti esemplificativi della moderna cultura decorativa viennese. Sale di promozione turistica, sale d'aspetto, ristorante e alloggio completo per il capostazione, testimoniavano non solo della validità del progetto complessivo, ma anche della validità di una "scuola decorativa" dove gli ideali artistici della Secessione avevano mirabilmente fruttificato sul terreno concreto dell'architettura, degli oggetti e delle materie lavorate nei laboratori delle Wiener Werkstätte ${ }^{45}$ (Figg. 20 e 21).

Prima di tratteggiare il quadro delle arti decorative al Parco Sempione, bisogna riferire del catastrofico incendio che due mesi dopo l'apertura, il 3 agosto, distrusse completamente il "Palazzo dell'arte decorativa" 46 (Figg. 22 e 23). Una "sciagura" che colpì soprattutto l'Italia e l'Ungheria e la cui portata si può immaginare dai lunghi accorati elenchi di espositori danneggiati e di oggetti perduti riportati dalle cronache del tempo. Il "Palazzo" fu ricostruito in forma più semplice (architetto Orsino Bongi) e le mostre si riaprirono dopo 40 giorni di febbrile lavoro: non così "magnifiche" come in precedenza, ma abbastanza fornite per dare un senso alle visite e per consentire reportages e commenti, che a volte intrecciavano il prima e il dopo in nodi quasi inestricabili per fissare la memoria e rendere il giusto omaggio agli espositori ${ }^{47}$.

44 Cfr. Ed. Ximenes, Il Padiglione del Belgio, in Milano e l'Esposizione Internazionale, cit., pp. 402-405 e 500-503; A. Pica, Il padiglione belga, cit., p.7.

45 Cfr. Le Mostre Austriache, in L'Esposizione illustrata di Milano, cit., 305-312; Ed. Ximenes, Il Padiglione dell'Austria, in Milano e l'Esposizione Internazionale, cit., 513-515.

46 Cfr. L'immane disastro e Le mostre perdute, in L'Esposizione illustrata di Milano, cit., 209-211, 211-212. L'incendio distrusse anche la Mostra del Duomo di Milano, la sezione dell'Architettura e la mostra dei "Lavori femminili". Per la mostra del Duomo (architetti Gaetano Moretti e Adolfo Zacchi), che perse preziosi documenti, cfr. E. Brivio, Il padiglione della Veneranda Fabbrica del Duomo all'Esposizione del 1906, in Milano verso il Sempione, cit., 238-239.

47 Cfr. Le nuove Gallerie dell'Arte Decorativa, in L'Esposizione illustrata di Milano, cit., 265-267. 
Tra le mostre incenerite e rapidamente riaperte con sforzi "immensi" era quella ungherese, allestita con la regia dello scultore e decoratore Géza Maróthi, i cui oggetti erano già stati ammirati a Torino nel 1902 e alla Biennale d'Arte di Venezia del 1905. A Milano l'Ungheria "abbagliava" ancora per la produzione fantasiosa e brillante, per la modernità che riusciva a riallacciarsi alla tradizione, per l'intenso afflato patriottico di un paese che vent'anni prima aveva celebrato il millennio e si era orgogliosamente messo sulla strada dell'indipendenza politica, culturale e artistica dal predominio austriaco ${ }^{48}$ (Figg. 24 e 25).

Accomunata all'Ungheria dalla medesima sventura stava l'Italia che, avendo la mostra più ampia in assoluto, fu la più danneggiata dal fuoco $^{49}$. Furono distrutti i magnifici merletti di Jesurum (Venezia); i 20 ambienti arredati della Fabbrica Italiana di Mobili (Milano), ivi compreso un salotto Impero "veramente magnifico" già acquistato dal Re; i mobili di Ducrot di Palermo; le sale di Valabrega (Torino); gli arredi di Grazioli e Gaudenzi, di Eugenio Quarti e dell'architetto Enrico Monti, tutti di Milano. Furono distrutte le vetrate artistiche di Giovanni Beltrami \& C. (Milano); i prodotti della "Società Ceramica di Laveno" e della ditta fiorentina Cantagalli; andò distrutto anche lo scalone in maiolica di Giovanni Palanti, un vero exploit fra tecnica ed arte, scultura e architettura, presentato dalla "Ceramica di Faenza", la gloriosa "industria artistica" che "dal Rinascimento era stata modello per l'intera Europa" $"$.

Alla riapertura si ripresentarono le ditte e gli artefici meglio organizzati, coloro che disponevano di ricchi campionari, di abbondante forza lavoro e di modelli facilmente replicabili, non comparvero più sia

48 Tra i numerosi resoconti, tutti positivi, cfr.: La nuova Mostra Ungherese, in L'Esposizione illustrata di Milano, cit., 283-284; A. Pica, La sezione ungherese, cit.; Ed. Ximenes, L'Ungheria all'Esposizione Internazionale di Milano, in Milano e l'Esposizione Internazionale, cit., 338-341; P. Chiesa, L'arte decorativa nella Esposizione di Milano. L'Ungheria, in "Arte Italiana Decorativa e Industriale", n. 9, settembre 1906, 69-75.

49 Le mostre perdute. Le Gallerie dell'Arte Decorativa Italiana, in L'Esposizione illustrata di Milano, cit., 211-214; M. B. Cerati, Le Arti Decorative Italiane e La nuova mostra d'Arte Decorativa Italiana, entrambi in Milano e l'Esposizione Internazionale, cit., 390-394 e 641-642.

50 Fra le arti decorative italiane. Una delle mostre perdute, in L'Esposizione illustrata di Milano, cit., 223-224. 
i pezzi unici e i tour de force tipici delle grandi esposizioni, sia i prodotti "speciali" dotati di un alto valore artistico e frutto di lavorazioni complesse, lunghe e accurate. Tra il ricordo degli oggetti distrutti e l'osservazione di quelli nuovi, tra il "fascino di un gentile compianto e la forza di una nobile ammirazione", i critici poterono tuttavia valutare abbastanza positivamente un'esposizione che rappresentava buona parte della produzione modernista italiana ${ }^{51}$.

Lo spazio più ampio era assegnato alle "arti del legno", ovvero a mobili e arredi in ragione del loro diretto rapporto con i bisogni quotidiani e di un tessuto produttivo ampiamente diffuso sul territorio nazionale, con particolare concentrazione nella regione. Rispetto alle espressioni più eccessive e stravaganti del Liberty o dell' "arte nuova" dei primissimi anni del secolo, il pittore Pietro Chiesa sulle pagine di "Arte Italiana Decorativa e Industriale" osservava un complessivo maggiore "ritegno", in accordo con la generale inclinazione europea che aveva abbandonato le "sagome ingombranti e assurde" e i superficiali allettamenti visivi a favore di linee più controllate e forme più sobrie. Si notava "un più abile ricorrere alle diverse proprietà" dei materiali e si apprezzava l'esecuzione accurata ${ }^{52}$.

Tra i migliori era l'ebanista milanese Eugenio Quarti, già noto in ambito internazionale per il prestigioso grand prix conquistato all'Exposition Universelle di Parigi del $1900^{53}$. Dal primo laboratorio fondato a Milano nel 1888 la sua attività si era sviluppata nel corso degli anni fino a superare la fase puramente artigianale e richiedere il lavoro di più 50 addetti. La ditta Quarti produceva pezzi raffinati, spesso destinati a una clientela esclusiva, ma era in grado di apprestare anche mobili più semplici ed economici e, soprattutto, di progettare arredi completi, grazie alla collaborazione di numerosi artisti e tecnici specialisti.

Alfredo Melani parlava di una "officina di mobili, ossia d'ambienti”, intendendo che l'attenzione del produttore moderno doveva

51 Fuoco distruttore, in "L'Artista Moderno", n. 16, 1906, 246.

52 P. Chiesa, L'arte decorativa all'Esposizione di Milano II. Arti del legno e dell'addobbo, in "Arte Italiana Decorativa e Industriale", n. 7, luglio 1906, 55.

53 Per l'opera di Eugenio Quarti (1867-1929) cfr. C. Alberici, R. Bossaglia, Eugenio e Mario Quarti. Dall'ebanista Liberty all'arredamento moderno, Milano, Comune di Milano, 1980; da ultimo Eugenio e Mario Quarti nelle raccolte del Castello Sforzesco, a cura di F. Tasso, Milano, Skira, 2008. 
andare oltre il singolo pezzo e pensare al mobile come parte di un sistema funzionale, simbolico e decorativo nella trama dello spazio domestico ${ }^{54}$. La raffinatezza del gusto, la sensibilità e l'accuratezza esecutiva, l'interesse per le nuove correnti estetiche, la volontà di creare mobili moderni senza rinunciare all'alta qualità ebanistica, erano i valori positivi di una produzione che aveva raggiunto una propria cifra espressiva, progressivamente depurata dai preziosismi decorativi dei primi anni di attività, per scoprire "il segreto della semplificazione" e della "signorile sobrietà" 55 .

Anche gli ambienti presentati originariamente da Quarti furono distrutti dall'incendio comportando una perdita di ben 60.000 lire $^{56}$, ma egli riuscì a sostituirli in gran parte con altri arredi, repliche e varianti già pronte in laboratorio o velocemente preparate per l'occasione. Difficile identificare esattamente le due fasi, per la disparità dei resoconti nei cataloghi ufficiali e nelle cronache giornalistiche; resta comunque evidenza di quattro ambienti completi: due camere da letto, piuttosto simili nel disegno, ma diverse per la scelta delle essenze, un salotto e una sala da pranzo, oggi conservata nelle Civiche Raccolte d'Arte ${ }^{57}$ (Figg. 26 e 27). Nell'insieme si caratterizzavano per un disegno pulito ed elegante, superfici piane, forme solide e squadrate, per gli accostamenti e gli accordi cromatici tra i legni, per gli intarsi e gli intagli attentamente distribuiti. Non a caso alcuni dei suoi pezzi furono scelti per essere riprodotti in disegni a scala 1:1 dalla rivista "Arte Italiana decorativa e industriale", diretta da Camillo Boito con il sostegno del Ministero di Agricoltura Industria e Commercio e principalmente destinata alle scuole di arti e mestieri, riconoscendone quindi le "intrinseche qualità didattiche" per la chiarezza e l'equilibrio della composizione ${ }^{58}$ (Fig. 28).

Tra i mobilieri a dimensione industriale si imponeva Vittorio Ducrot di Palermo, geniale imprenditore che, nell'arco di circa un ventennio, aveva trasformato una piccola impresa artigianale in un complesso produttivo con circa 200 operai e fornito di aggiornati dispositivi

54 Cfr. A. Melani, Eugenio Quarti ebanista, in "Arte Italiana Decorativa e Industriale”, n. 2, febbraio 1904, 12-16, la citazione è a p. 13.

55 Ibidem.

56 Fuoco distruttore, cit. 247.

57 Cfr. Eugenio e Mario Quarti nelle raccolte, cit., 47-49.

58 Cfr. "Arte Italiana Decorativa e Industriale", 1906, Dettagli nn. 42, 43, 44. 
meccanici, Nel 1899 egli aveva chiamato a collaborare alla parte artistica l'architetto Ernesto Basile che da una formazione tardo eclettica aveva aderito alle istanze moderniste ${ }^{59}$. Insieme per circa un decennio, Ducrot e Basile avevano dato vita a un fecondo sodalizio professionale tra industria e design che contribuì largamente al successo e alla qualità dei prodotti.

I buoni frutti si videro anche a Milano dove, a seguito dell'incendio di agosto, la Ducrot riuscì a ricostruire in tempi assai brevi la propria mostra in virtù della sua efficiente organizzazione industriale ${ }^{60}$. Per la maggior parte firmati da Ernesto Basile, gli arredi Ducrot dimostravano agli occhi dei critici che poteva esserci qualità nel coesistere di antico e moderno, come nell'incontro fra innovazione e tradizione; venivano così valutati positivamente $\mathrm{i}$ mobili liberamente ispirati allo stile Luigi XVI o allo stile Impero (allora "tornati di moda") che guardavano alla Francia e alla sua squisita eleganza anticipando un ritorno al neoclassicismo proprio del decennio seguente.

Si apprezzavano parimenti i pezzi più moderni contrassegnati da linee diritte e da un'attenta costruzione geometrica, i mobili economici creati sia per le case più modeste, sia per le forniture di albergo, ma soprattutto si parlava con favore di una serie di poltroncine, chaise longue e tavolini da "giardino o da veranda", denominata "carretto siciliano" per la vivace policromia e l'ascendenza dall'arte popolare regionale. Si trattava di arredi poco costosi, votati all'uso, semplici e di linea essenziale che, proprio per questo, presupponevano "un'eccellenza minuziosa, abile e paziente [...], giacché in essi ogni piccolo difetto di proporzione, ogni piccola trascuraggine [sic] di fattura salta subito agli occhi” ${ }^{61}$ (Fig. 29).

Almeno un accenno è dovuto ai protagonisti nei settori dei vetri artistici e dei ferri battuti, generalmente apprezzati nelle esposizioni come ambiti italiani di eccellenza. Nel primo l'esempio era offerto dall'Officina di Vetri Artistici G. Beltrami \& C. dove, sotto la direzione

59 Per la Ducrot di Palermo e i mobili disegnati da Ernesto Basile, cfr. tra i numerosi studi di E. Sessa: Mobili e arredi di Ernesto Basile nella produzione Ducrot, Palermo, Novecento, 1981; Id., Ernesto Basile: dall'eclettismo classicista al modernismo, Palermo, Novecento, 2002. $131-143$

60 Cfr. La casa Ducrot di Palermo, in "L'Artista Moderno", 10 maggio 1907,

61 V. Pica, La sezione italiana I, cit, 254. 
di Giovanni Beltrami, erano attivi gli artisti Giovanni Buffa, Innocente Cantinotti, Guido Zuccaro, autori di vetrate che arricchirono gli ambienti di molte importanti architetture del periodo ${ }^{62}$. Pesantemente danneggiato dall'incendio (andarono perse tutte le opere, per lo più già vendute) Beltrami si ripresentò con altri esemplari di vetri istoriati caratterizzati da una "robusta figurazione plastica" e una "gioconda vivacità di tinte" che valorizzavano le potenzialità del materiale in termini di luci, colori e contorni, giocando sull'arabesco, il raggruppamento delle forme, gli audaci rapporti cromatici ${ }^{63}$ (Fig. 31).

"Vitalità" e "vigore" erano categorie sempre associate all'opera di Alessandro Mazzucotelli, maestro indiscusso nell'arte del ferro battuto, per indicarne sia l'ispirazione dalla natura, sia la forza del gesto nel piegare alla decorazione la dura materia. I suoi cancelli, ringhiere, inferriate e lampade presentavano quei tratti larghi e maschi che parevano derivare dalla "forza bruta del ferro" e seguivano i modelli di un mondo naturale osservato con amore e attenzione per riprodurne veristicamente lo svolgersi e, nello stesso tempo, per adottarne le leggi, i fondamenti geometrici e l'impianto strutturale ${ }^{64}$.

Mazzucotelli aveva presentato lavori in diverse sezioni, partecipando al decoro complessivo di ambienti e padiglioni. Non tutte le sue opere andarono quindi distrutte dal fuoco e tra queste risaltava nel Padiglione degli Orafi lo straordinario "cancello dei gladioli" dove si poteva osservare una trama ordinata di pieni e vuoti, di studiati equilibri tra le parti più pesanti collocate alla base e quelle più leggere alla sommità, tra l' "ordinata libertà" dei rami fioriti e la solidità dei nodi, delle sbarre e dei motivi a scacchiera (Fig. 32).

62 Giovanni Beltrami (1860-1926) fu pittore, imprenditore, giornalista, presidente dell'Accademia di Brera, direttore della casa editrice Treves: cfr. E. Fezzi, Beltrami, Giovanni, in Dizionario Biografico degli Italiani, Roma, Istituto della Enciclopedia Italiana, vol. 8, 1966, ad vocem. Notizie specifiche in: A. Novellone, Il Liberty nell'arte della vetrata a Milano ai primi del '900: la Ditta G.Beltrami e C. Vetrate Artistiche, in "Storia dell'Arte", n. 62, 1988, 87-95; La vetrata Liberty a Milano, Catalogo della mostra, a cura di C. Pirina, M.P. Bassi, E. Brivio, Milano, Scotti, 1990, passim.

63 Citazioni da Vittorio Pica, La sezione italiana II, cit., 330.

64 Ibidem, pp. 330-332. Cfr. inoltre A. Mazzucotelli, I ferri battuti, prefazione di U. Ojetti, Milano, Bestetti e Tumminelli, 1912; R. Bossaglia, A. Hammacher, Mazzucotelli, l'artista italiano del ferro battuto liberty, Milano, Il Polifilo, 1971; R. Ruscio, Mazzucotelli, Alessandro, in Dizionario Biografico degli Italiani, Roma, Istituto della Enciclopedia Italiana, 72, 2008, ad vocem. 
Giovanni Buffa, della G. Beltrami \& C., e Alessandro Mazzucotelli insegnavano anche nelle "Scuole-laboratorio di arte applicata all'industria" fondate nel 1903 dalla Società Umanitaria e comprendenti sezioni per "fabbri ornatisti, ebanisti, intagliatori, intarsiatori, tappezzieri, orefici, incisori, cesellatori, decoratori murali, verniciatori, doratori, pittori di insegne, lavoranti nel vetro artistico" ${ }^{65}$. Si tratta, come è noto, di un'istituzione fra le più interessanti e meritevoli di attenzione, 'artefice' di progetti e realizzazioni che consentono di gettare un ponte tra l'Esposizione del 1906 e gli eventi del dopoguerra e che meritano almeno un accenno conclusivo.

All'Esposizione l'Umanitaria disponeva di un padiglione autonomo al parco Sempione: una costruzione in legno, un'architettura 'rapida', leggera, semplice e priva di ostentazione ${ }^{66}$. All'interno si trovavano la documentazione sulle numerose iniziative a sostegno delle classi lavoratrici, gli elaborati degli studenti delle scuole professionali e di arte applicata all'industria, nonchè la ricostruzione di due stanze arredate nel quartiere operaio di via Solari. Fedelmente all'assunto di fornire ai più deboli i mezzi per il loro miglioramento, le scuole erano il campo più strategico di intervento e si qualificavano per l'obiettivo esplicito di rinnovare metodi e strumenti, spazi e attrezzature, materie e insegnamenti.

Il piccolo padiglione non fu tra i più visitati, ma i suoi effetti sulle arti decorative furono assai più duraturi delle circostanze transitorie della mostra, allo stesso modo in cui l'Umanitaria fu continuamente attiva nel rilancio delle arti e dell'insegnamento artistico-industriale. Il suo impegno continuò infatti per tutto il Novecento con iniziative sempre notevoli, tra cui nel 1919, alla fine della guerra, l'Esposizione Regionale Lombarda di Arti Decorative, intesa come una prima rassegna periodica che avrebbe contribuito "al rinnovamento delle industrie

65 Cfr. nell'abbondante bibliografia sull'istituzione: L'opera della Società Umanitaria dalla sua fondazione ad oggi, Milano, Scuola del Libro, 1906; L'azione dell'Umanitaria per l'istruzione professionale operaia, Milano, Cooperativa Grafica degli Operai, 1921; Società Umanitaria, L’Umanitaria e la sua opera, Cooperativa Grafica degli Operai, Milano 1922; R. Bauer, La Società Umanitaria. Fondazione P.M. Loria 1893-1963, Pizzi, Milano 1964; E. Decleva, Etica del lavoro, socialismo, cultura popolare: Augusto Osimo e la Società Umanitaria, Milano, Angeli, 1985.

66 Cfr. O. Selvafolta, La Società Umanitaria all'Esposizione del 1906 e il rinnovamento delle arti applicate, in "Archivio Storico Lombardo", serie XII, 9; 2005-2006, $105-145$. 
artistiche nel paese [...], e a stimolare più intimi rapporti fra industriali, artefici e artisti e fra essi e il pubblico" ${ }^{67}$.

La mostra fu anche l'occasione per rimeditare sulle passate esperienze e diede impulso, nel 1922, alla fondazione, nella Villa Reale di Monza, dell'Università delle Arti Decorative (dal 1928 denominata Istituto Superiore per le Industrie Artistiche) promossa dal Consorzio Milano-Monza-Umanitaria, con l'obiettivo di fungere da centro di raccordo per le molteplici declinazioni del sapere connesse ai mestieri dell'arte ${ }^{68}$. L'inizio dei corsi, nel 1923, coincise con l'avvio di un programma espositivo sistematico e di largo respiro, ovvero con la prima Esposizione Biennale di Arti Decorative Moderne di Monza. Nel 1933 la $\mathrm{V}$ edizione si aprirà a Milano, con il nome di Triennale, nell'appena costruito Palazzo dell'Arte al Parco Sempione: quasi un luogo elettivo per quelle arti decorative, industriali o applicate che si erano faticosamente "affermate" come parti significative della cultura del progetto nei grandi eventi espositivi del secolo precedente e che qui troveranno ulteriori spazi di elaborazione e riconoscimento.

67 C. Bozzi, Discorso inaugurale, in Esposizione Regionale Lombarda d'Arte Decorativa. Società Umanitaria, settembre-ottobre 1919, Milano, Alfieri \& Lacroix, 1919, s.n.p. Cfr. anche Esposizione Regionale Lombarda d'Arte Decorativa. Settembreottobre 1919. Catalogo illustrato, Cooperativa Grafica degli Operai, Milano 1919.

68 Cfr. L'Università delle Arti Decorative del Consorzio Milano-MonzaUmanitaria retta dalla Società Umanitaria nella Villa Reale di Monza, Milano, Cooperativa Grafica degli Operai, 1922. 


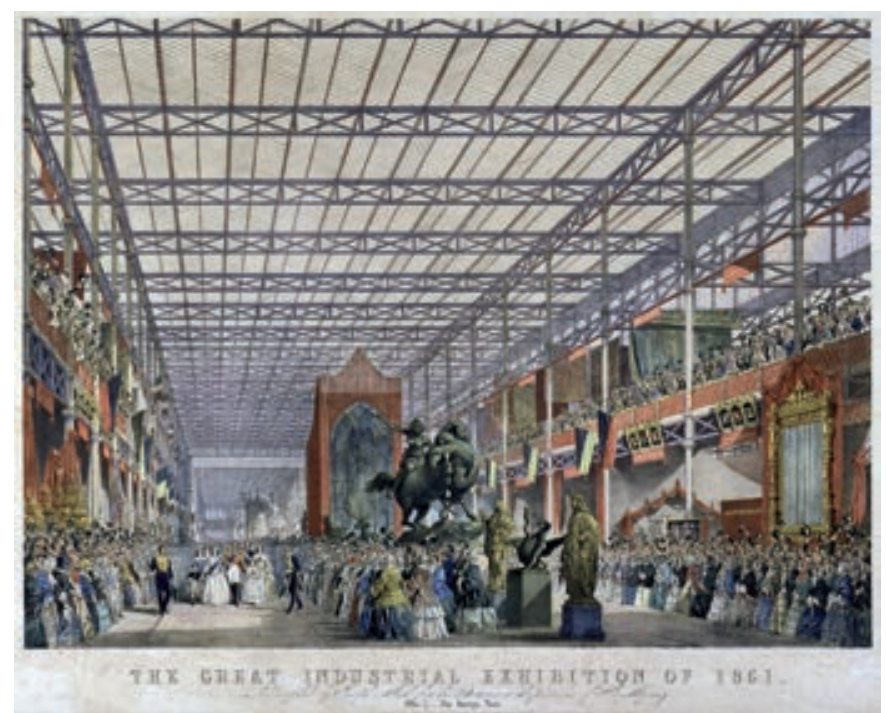

Fig. 1. The Great Industrial Exhibition nel Crystal Palace di Londra, 1851. Litografia di Joseph Nash. Immagine di public domain.

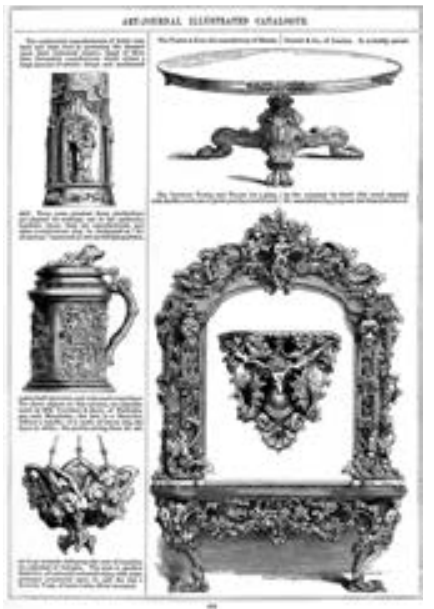

Fig. 2. Pagina di The Art Journal Illustrated Catalogue. The Industry of All Nations, London, George Virtue, 1851.
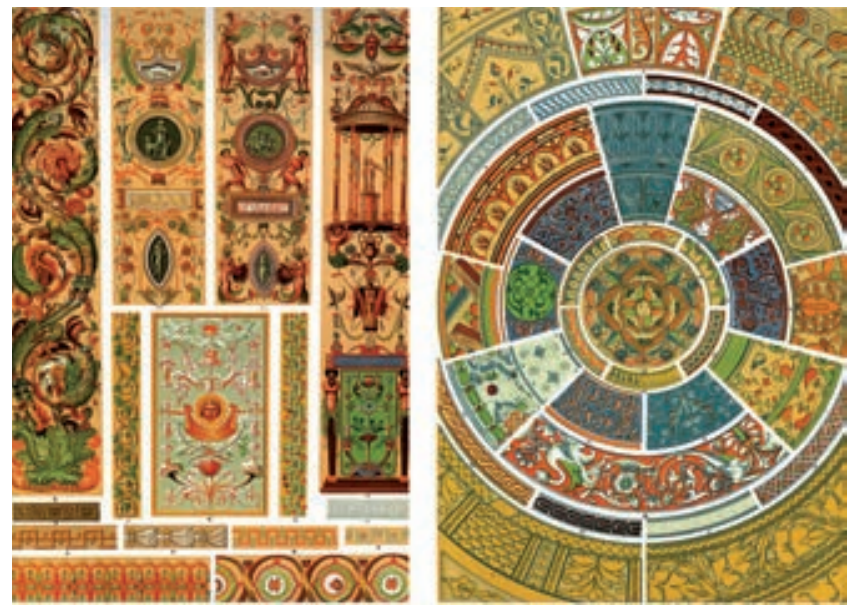

Fig. 3. Pagine della Grammar of Ornament by Owen Jones, London, Day \& Son, 1856. 


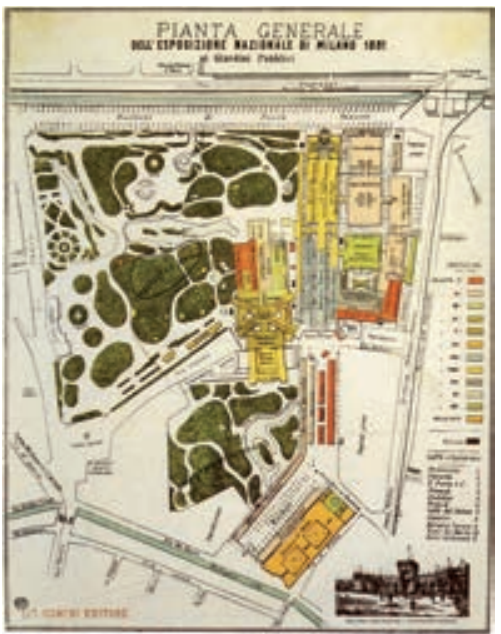

Fig. 4. Pianta generale dell'Esposizione Nazionale di Milano 1881 ai Giardini Pubblici. Milano, Civica Raccolta delle Stampe Achille Bertarelli.

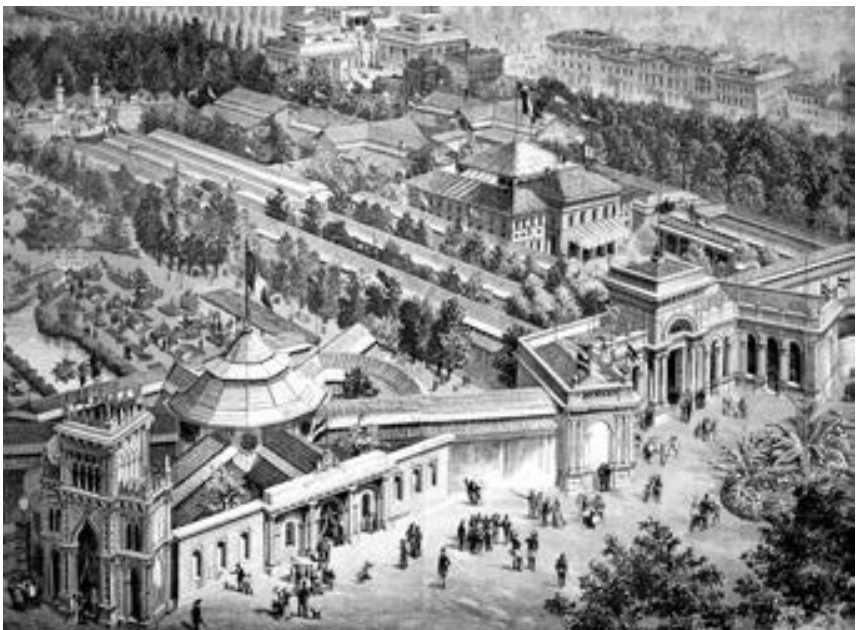

Fig. 5. Veduta a volo d'uccello della Esposizione Nazionale di Milano 1881 ai Giardini Pubblici. Da Milano e l'Esposizione Italiana del 1881, Milano, Treves, 1881.

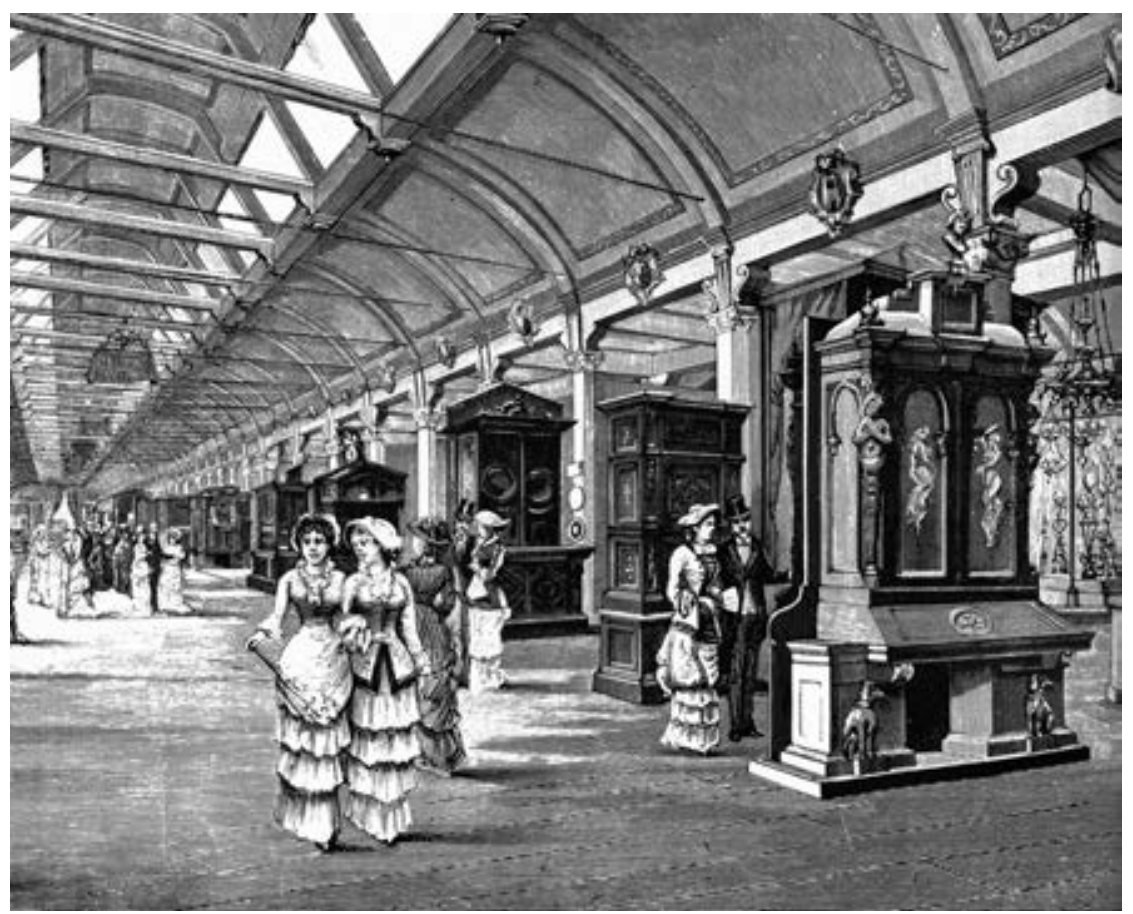

Fig. 6. Veduta della "Galleria centrale dei mobili artistici" all'Esposizione del 1881. Da L'Esposizione Italiana del 1881 in Milano, Milano, Sonzogno, 1881. 


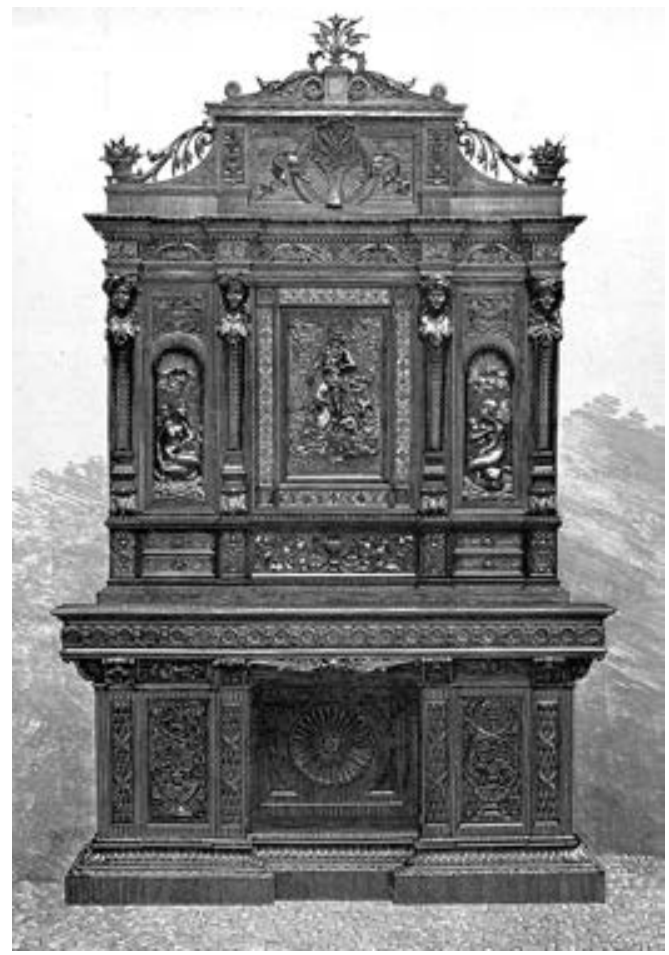

Fig. 7. Stipo di Pietro Zaneletti all'Esposizione del 1881.

Da L'Esposizione Italiana del 1881 in Milano, Milano, Sonzogno, 1881.

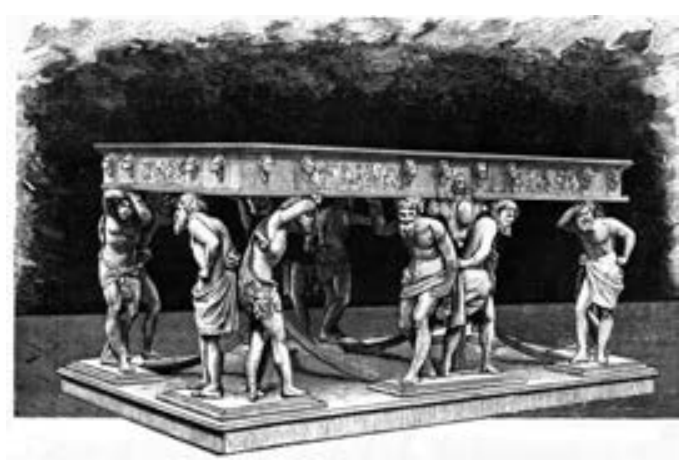

Fig. 8. Tavolo di Francesco Bortolotti all'Esposizione del 1881. Da L'Esposizione Italiana del 1881 in Milano, Milano, Sonzogno, 1881.

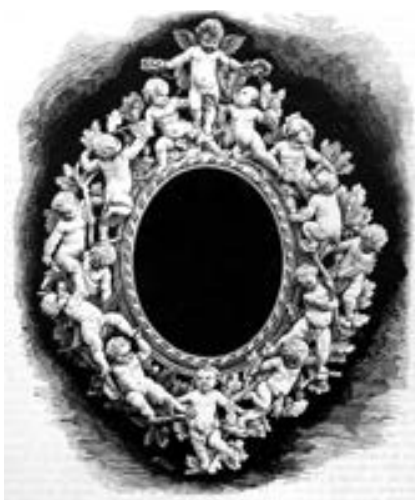

Fig. 9. Specchio intagliato di Valentino Panciera Besarel all'Esposizione del 1881.

Da L'Esposizione Italiana del 1881 in Milano, Milano, Sonzogno, 1881. 


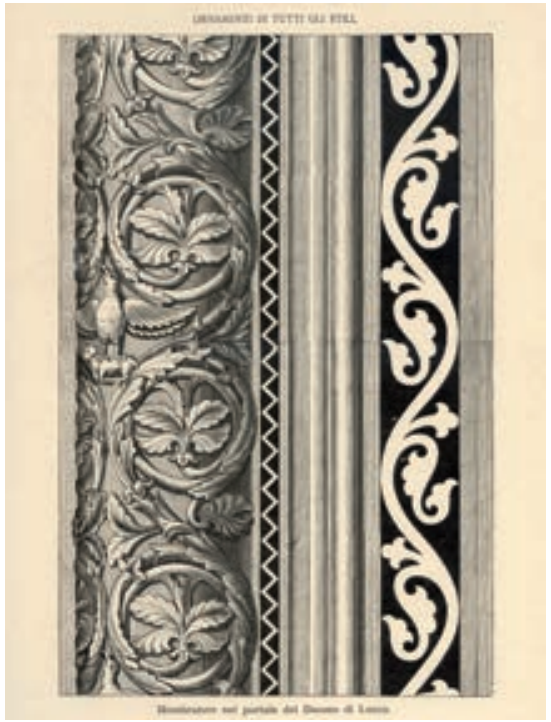

Fig. 10. Membrature nel portale del Duomo di Lucca in Camillo Boito, Ornamenti di tutti gli Stili, Milano, Hoepli, 1881.

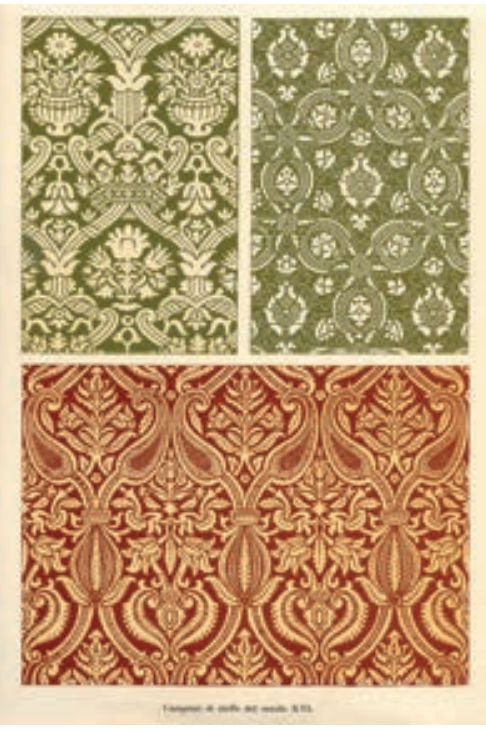

Fig. 11. Campioni di Stoffe del secolo XVI in Camillo Boito, Ornamenti di tutti gli Stili, Milano, Hoepli, 1881.

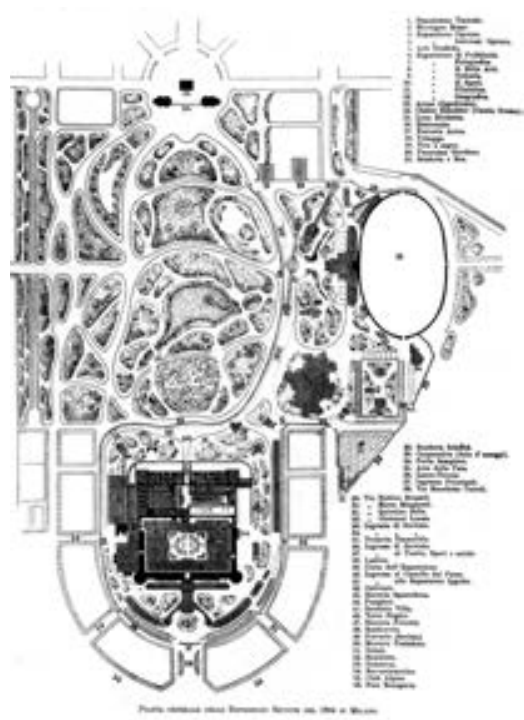

Fig. 12. Pianta generale delle Esposizioni Riunite del 1894 al Parco Sempione di Milano. Da Le Esposizioni Riunite di Milano 1894. Milano, Sonzogno, 1894.

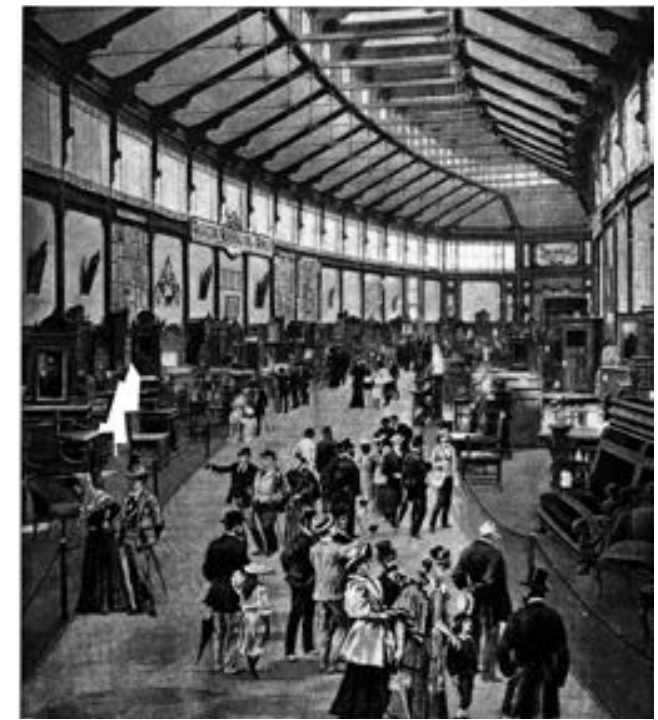

Fig. 13. La galleria generale del Mobilio alle Esposizioni Riunite del 1894.

Da Le Esposizioni Riunite di Milano 1894. Milano, Sonzogno, 1894. 


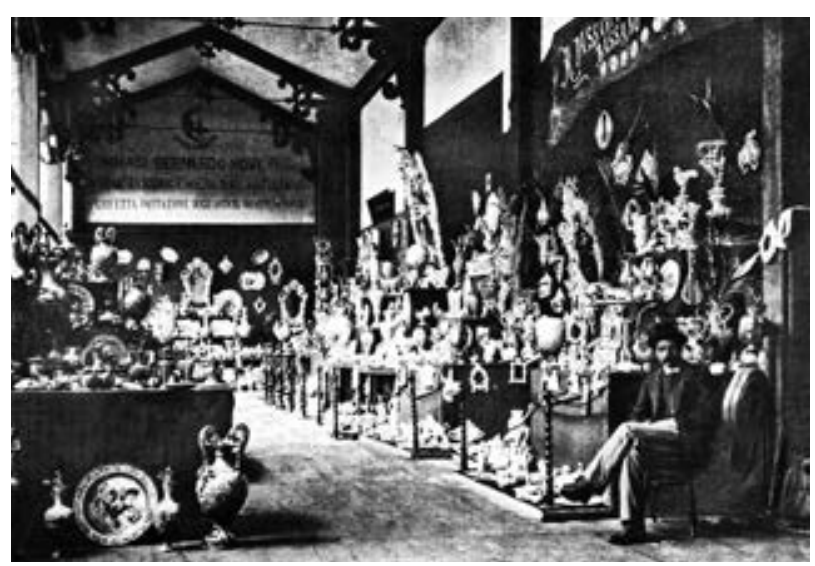

Fig. 14. Ceramiche "perfette imitazioni degli antichi smalti di Nove" nella Mostra Operaia alle Esposizioni Riunite del 1894. $\mathrm{Da}$ Le Esposizioni Riunite di Milano 1894. Milano, Sonzogno, 1894.

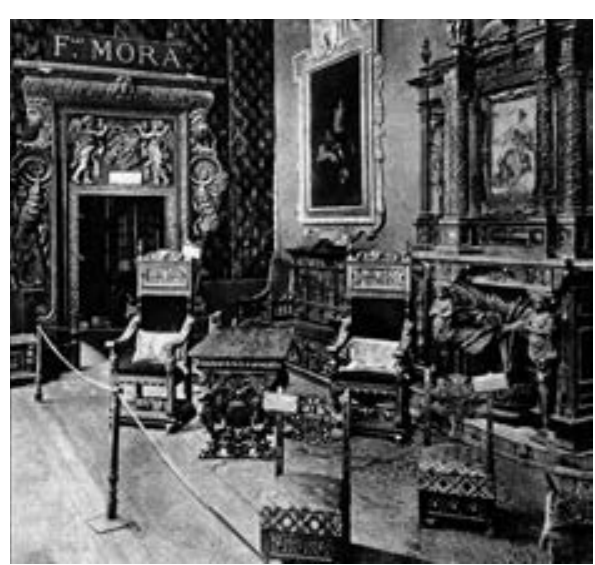

Fig. 15. Salotto dei Fratelli Mora alle Esposizioni Riunite del 1894.

Da Le Esposizioni Riunite di Milano 1894. Milano, Sonzogno, 1894.
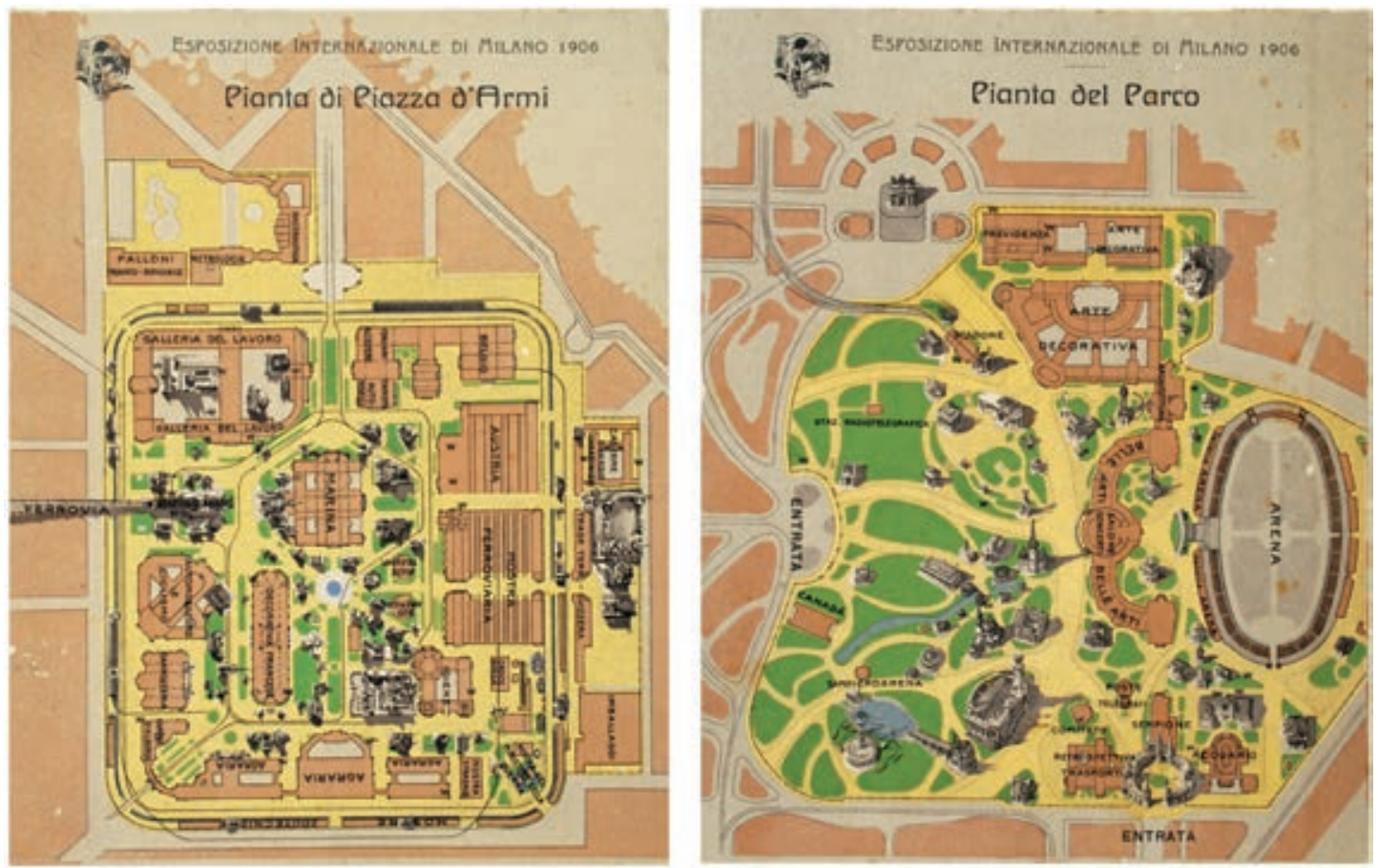

Fig. 16. Piante dell'Esposizione Internazionale di Milano 1906, in piazza d'Armi e nel Parco Sempione. Milano, Civica Raccolta delle Stampe Achille Bertarelli. 


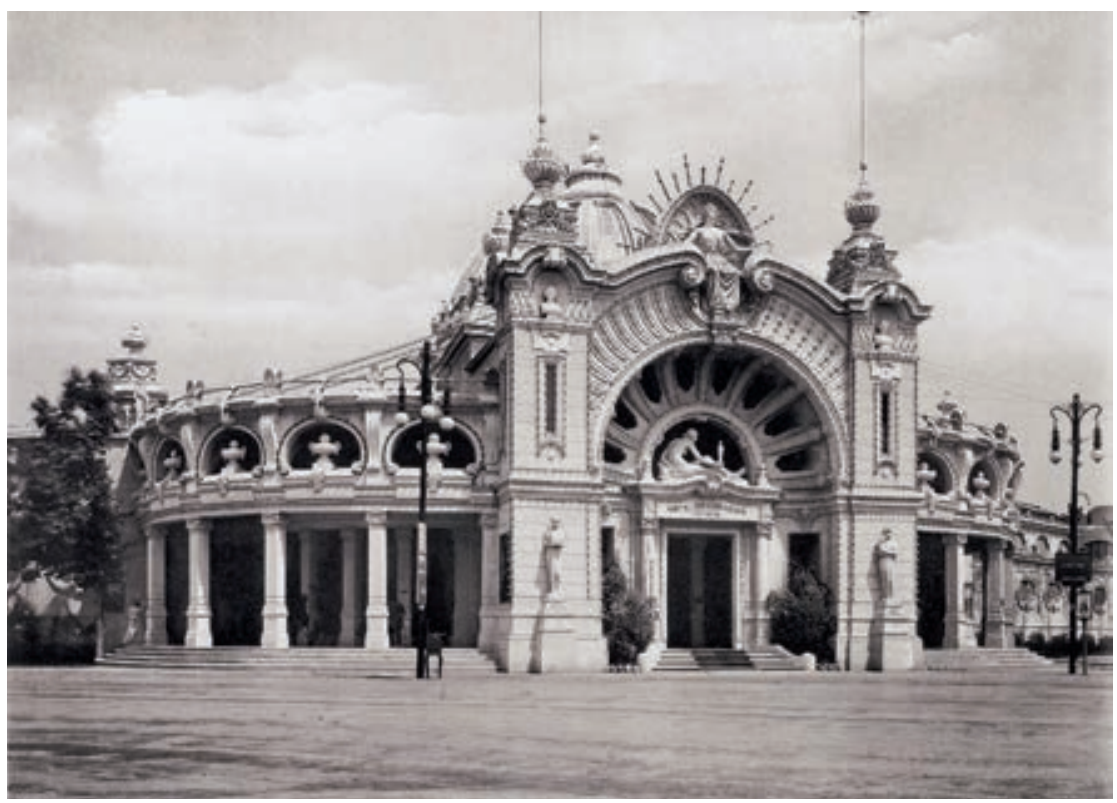

Fig. 17. Padiglione dell'Arte Decorativa al Parco Sempione, architetto Sebastiano Locati. Da "L'Edilizia Moderna", 1906.

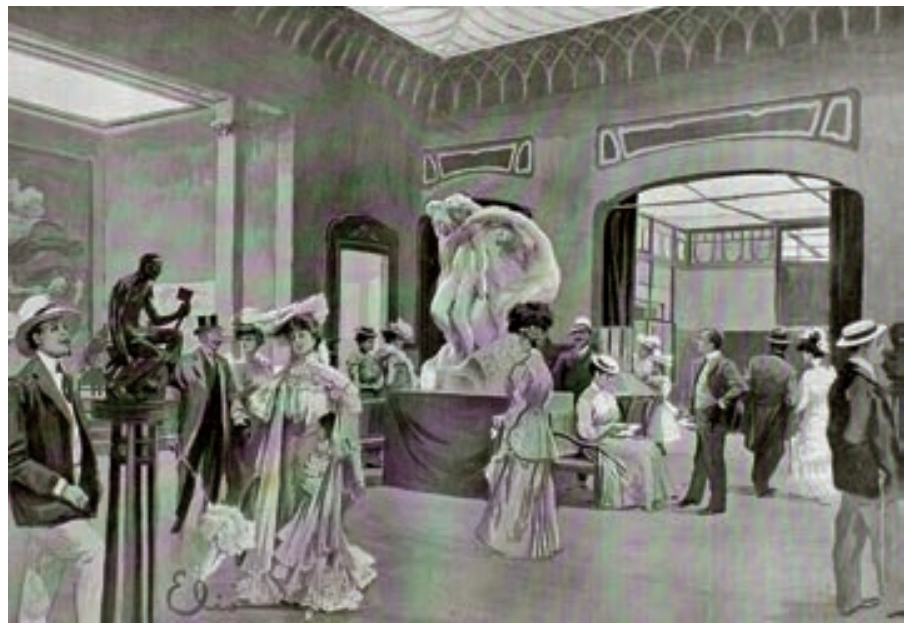

Fig. 18. Padiglione del Belgio, "Sala Horta".

Da Milano e l'Esposizione Internazionale del Sempione 1906, Milano, Treves, 1906.

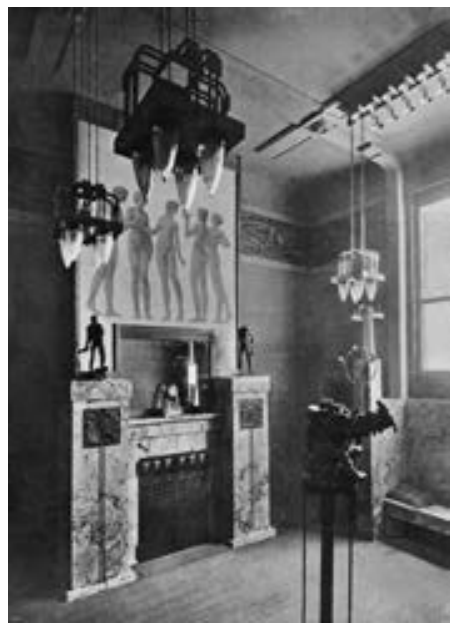

Fig. 19. Padiglione del Belgio, salotto presentato da Léon Sneyers.

Da Milano e l'Esposizione Internazionale del Sempione 1906, Milano, Treves, 1906. 


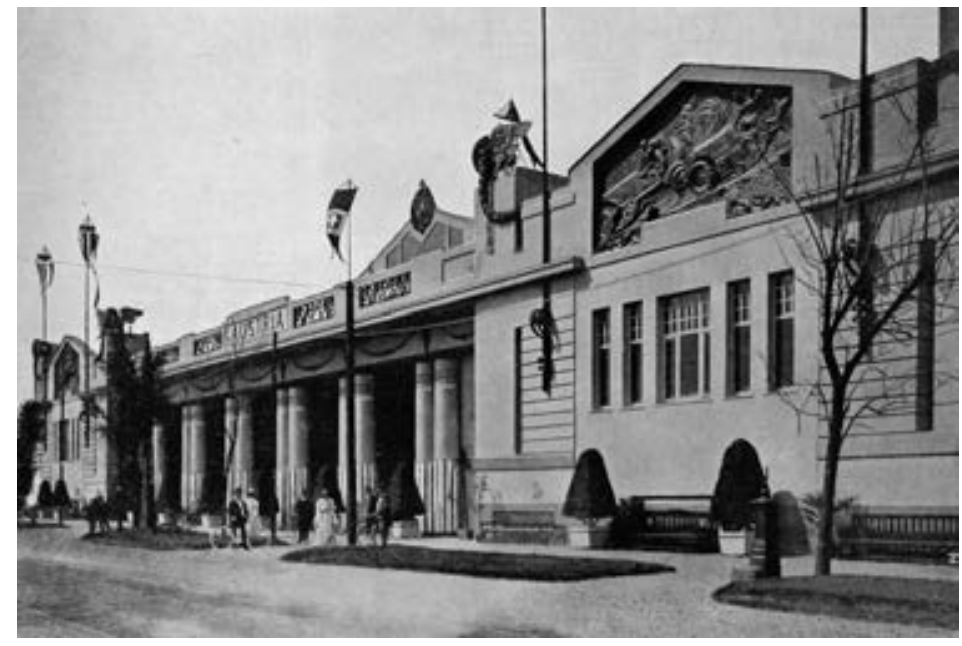

Fig. 20. Padiglione dell'Austria, architetto Ludwig Baumann.

Da L'Esposizione illustrata di Milano 1906, Milano, Sonzogno, 1906.
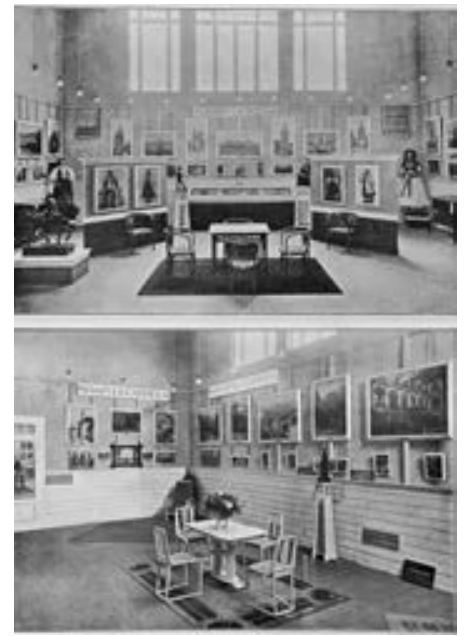

Fig. 21. Padiglione dell'Austria, Sale della Boemia e della Carniola (Slovenia)

Da Milano e l'Esposizione Internazionale del Sempione 1906, Milano, Treves, 1906.

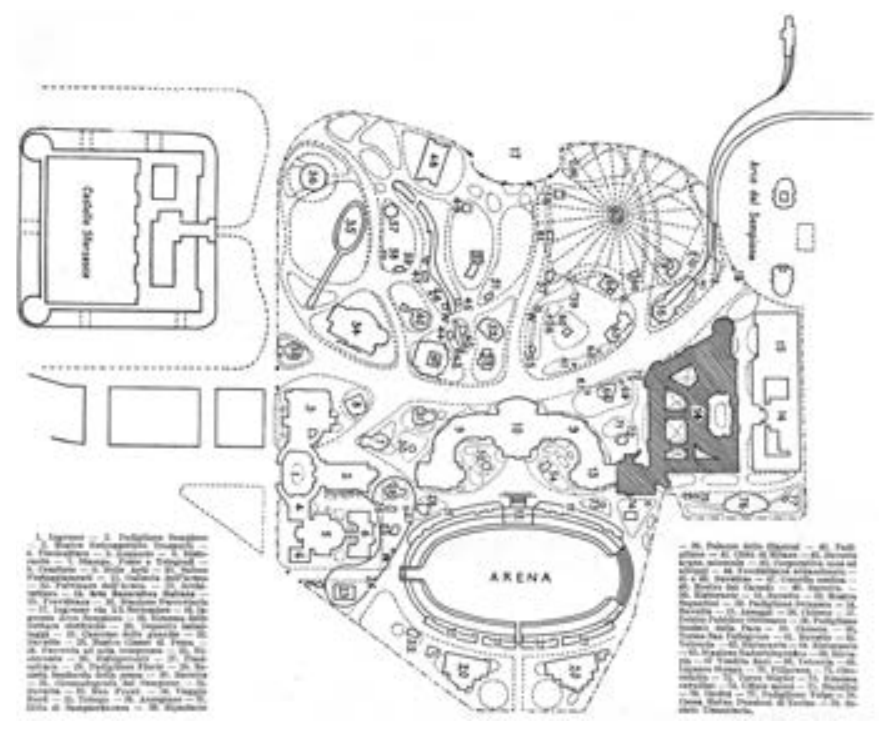

Fig. 22. Pianta dell'Esposizione al Parco Sempione, a tratteggio le parti distrutte dall'incendio. Da Milano e l'Esposizione Internazionale del Sempione 1906, Milano, Treves, 1906.

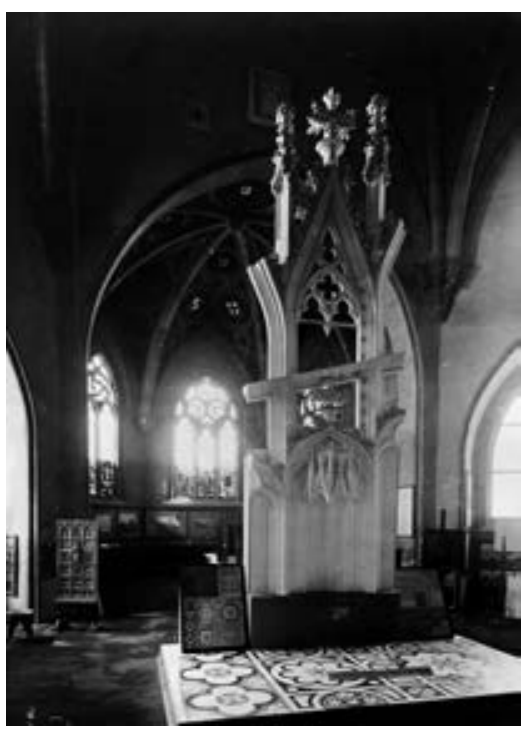

Fig. 23. La mostra della Veneranda Fabbrica del Duomo di Milano, distrutta dall'incendio. Milano, Archivio della Veneranda Fabbrica del Duomo. 


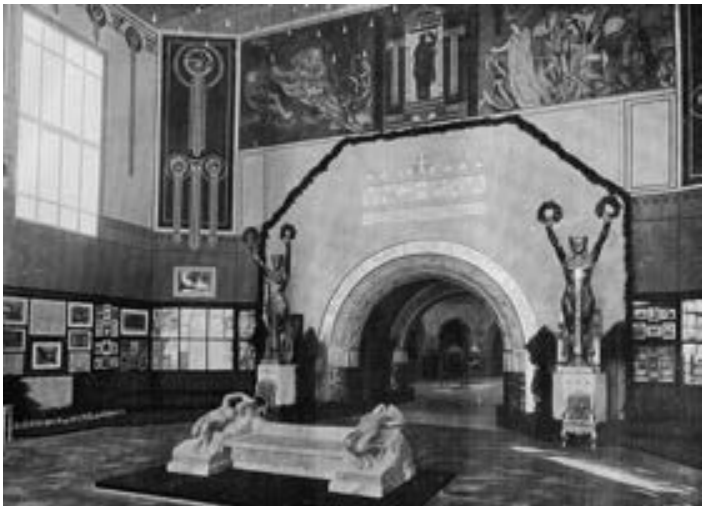

Fig. 24. Ingresso alla mostra dell'Ungheria ricostruita dopo l'incendio. Da Milano e l'Esposizione Internazionale del Sempione 1906, Milano, Treves, 1906.

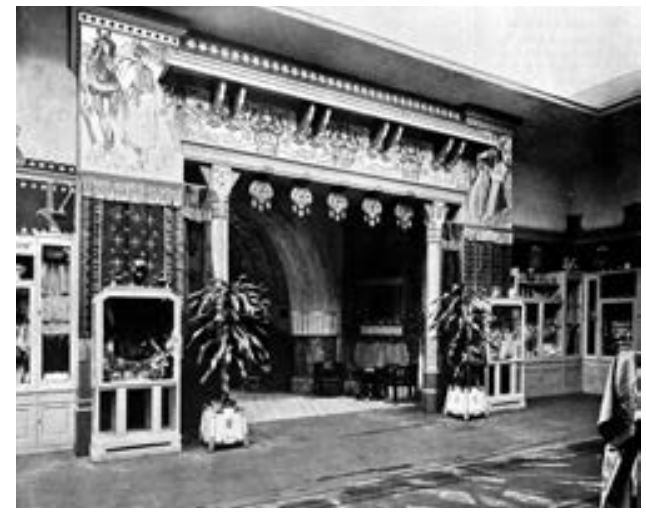

Fig. 25. Un ambiente della mostra delle Arti Decorative Ungheresi, ricostruita dopo l'incendio. Da Milano e l'Esposizione Internazionale del Sempione 1906, Milano, Treves, 1906.

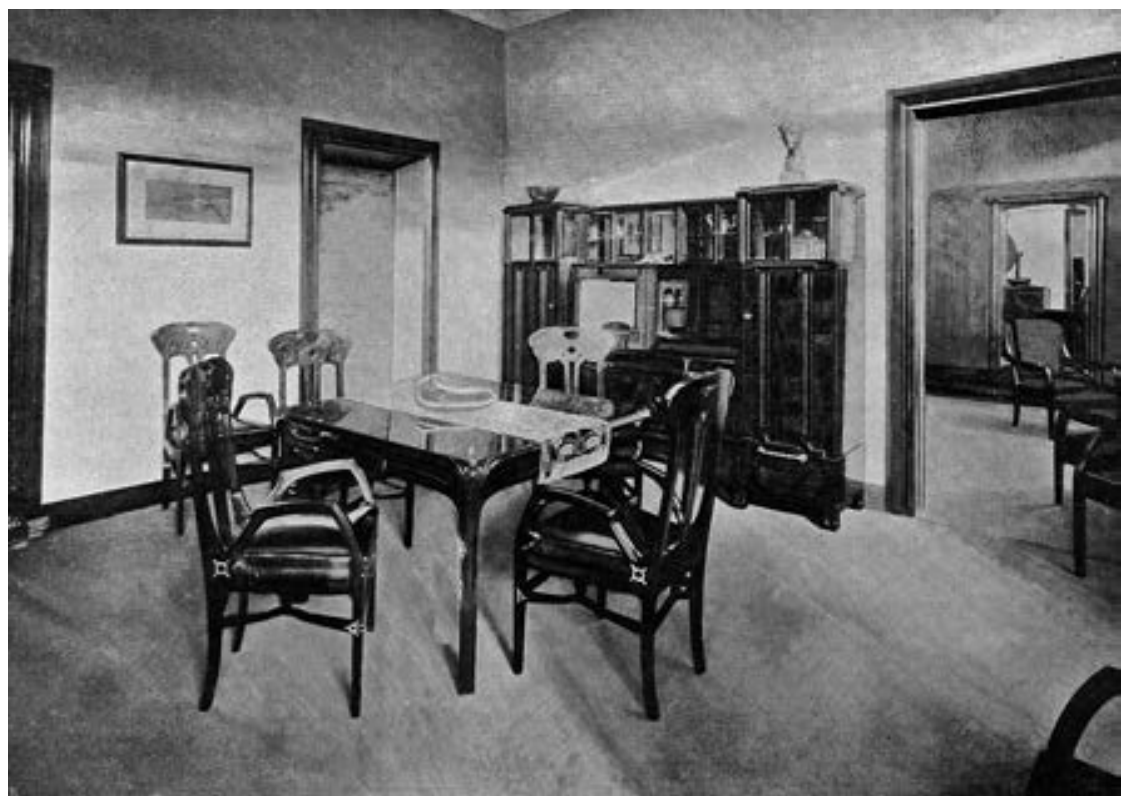

Fig. 26. Sala da pranzo presentata dall' ebanista Eugenio Quarti alla mostra delle Arti Decorative Italiane dopo l'incendio. Da Milano e l'Esposizione Internazionale del Sempione 1906, Milano, Treves, 1906. 


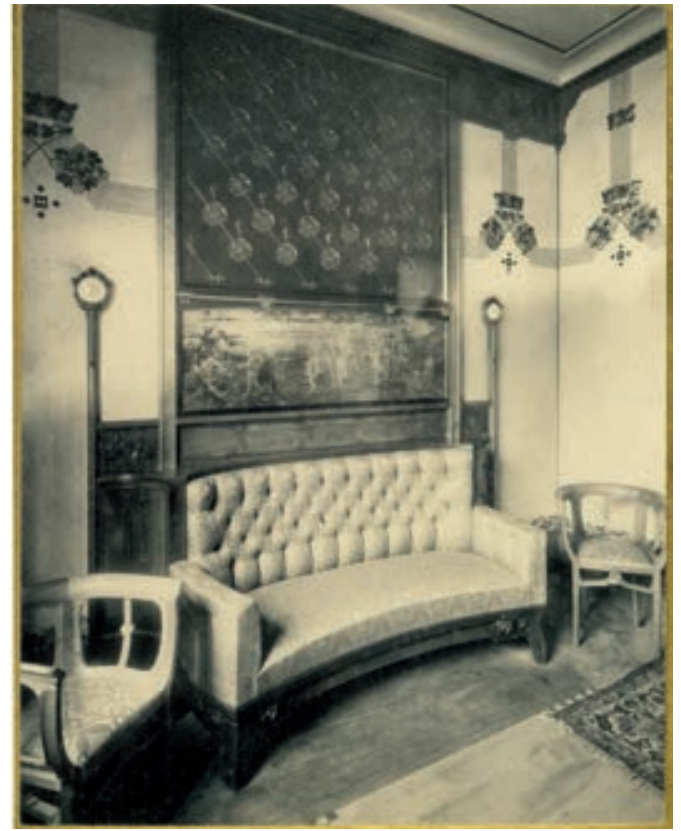

Fig. 27. Salottino presentato da Eugenio Quarti, vincitore del Gran Premio Reale dell'Esposizione. Milano, Civica Raccolta delle Stampe Achille Bertarelli, Fondo Eugenio Quarti.

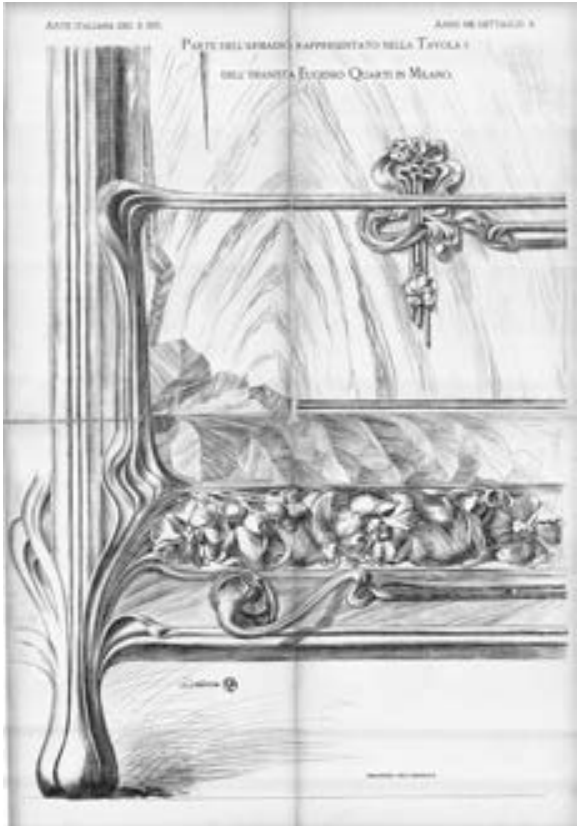

Fig. 28. Dettagli in scala 1:1 di mobili di Eugenio Quarti. Da "Arte Italiana Decorativa e Industriale”, 1906.

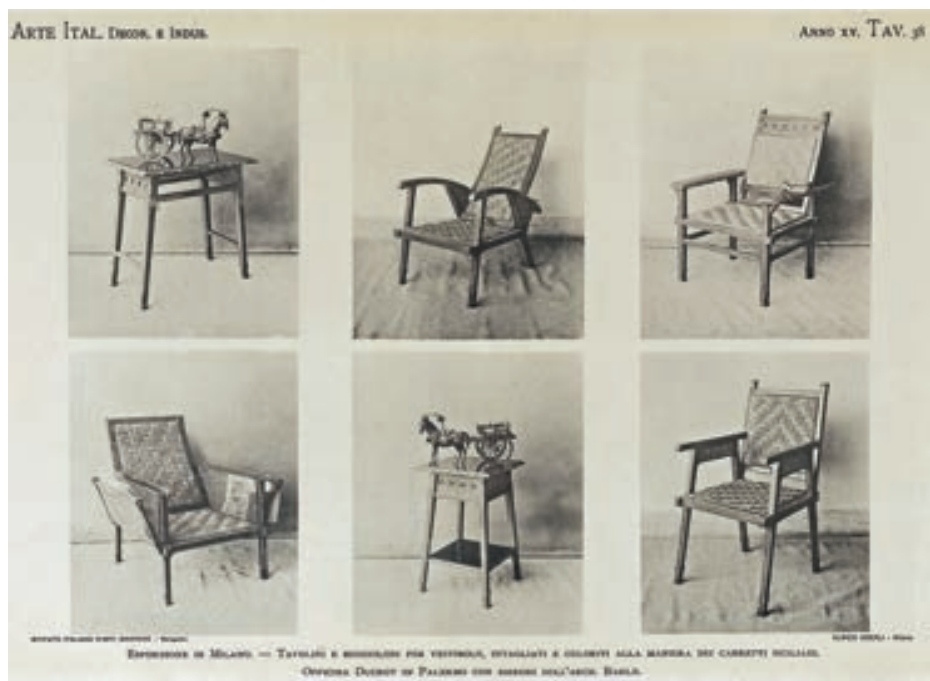

Fig. 29. Mobili della serie "carretto siciliano" disegnati dall'architetto Ernesto Basile presentati dalla ditta Ducrot di Palermo.

Da "Arte Italiana Decorativa e Industriale", 1906. 


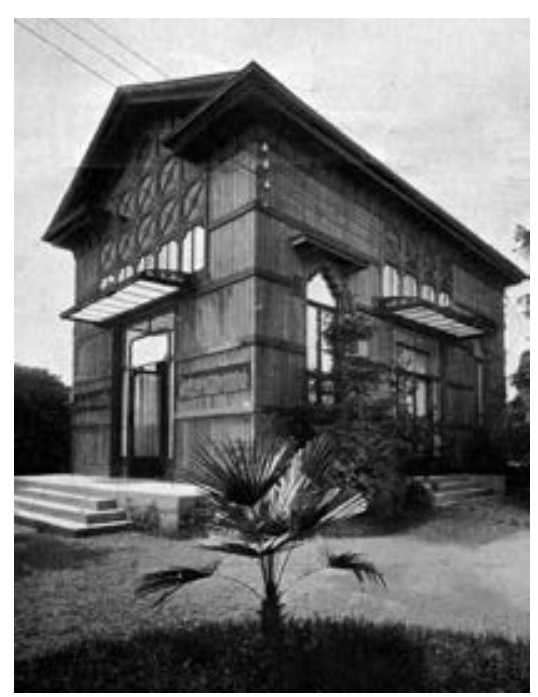

Fig. 30. Padiglione della Società Umanitaria al Parco Sempione, architetto Luigi Conconi.

Da L'Esposizione illustrata di Milano 1906, Milano, Sonzogno, 1906.

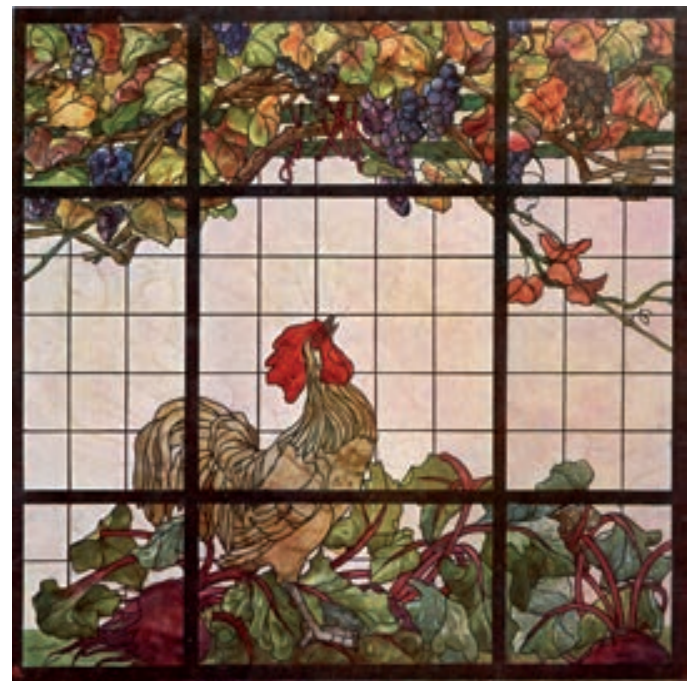

Fig. 31. Vetrata disegnata da Giovanni Buffa, direttore della Scuola del vetro della Società Umanitaria. Da "Modelli d'Arte Decorativa", 1910.

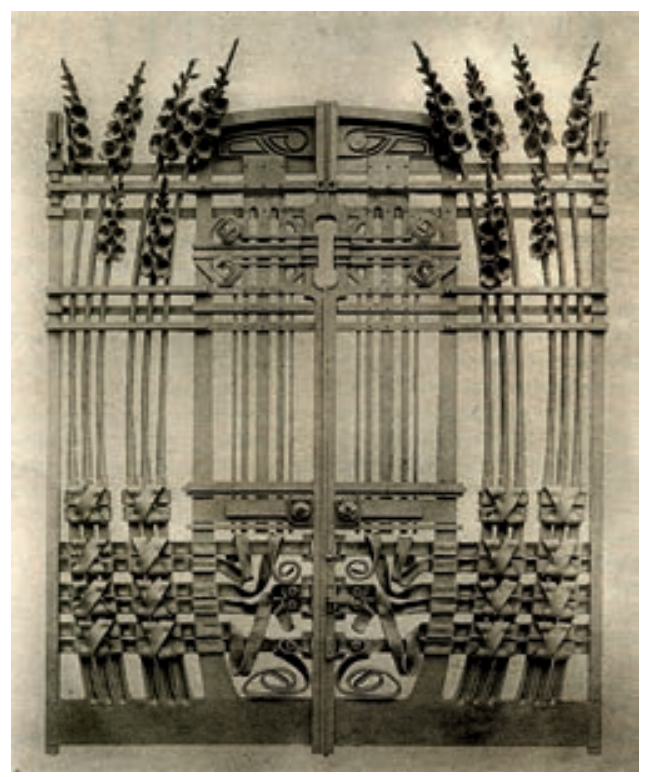

Fig. 32. Cancello dei gladioli, presentato da Alessandro Mazzucotelli nel Padiglione degli Orafi al Parco Sempione. Da A. Mazzucotelli, i ferri battuti, Milano, Bestetti e Tumminelli, 1912. 